\title{
Activité du manager de réception à l'épreuve de la digitalisation : cas d'un projet d'accueil en mobilité dans une chaîne hôtelière internationale
}

Activity of the front office reception manager in the challenge of the digitalization: case of a project of reception in mobility in an international hotel chain

Pierre Flandrin, Cécile van de Leemput et Catherine Hellemans

\section{OpenEdition}

Journals

Édition électronique

URL : https://journals.openedition.org/activites/6760

DOI : 10.4000/activites. 6760

ISSN : $1765-2723$

Éditeur

ARPACT - Association Recherches et Pratiques sur les ACTivités

Référence électronique

Pierre Flandrin, Cécile van de Leemput et Catherine Hellemans, « Activité du manager de réception à l'épreuve de la digitalisation : cas d'un projet d'accueil en mobilité dans une chaîne hôtelière internationale », Activités [En ligne], 18-2 | 2021, mis en ligne le 15 octobre 2021, consulté le 07 avril 2022. URL : http://journals.openedition.org/activites/6760 ; DOI : https://doi.org/10.4000/activites 6760

Ce document a été généré automatiquement le 7 avril 2022.

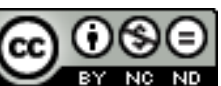

Activités est mis à disposition selon les termes de la licence Creative Commons Attribution - Pas d'Utilisation Commerciale - Pas de Modification 4.0 International. 


\section{Activité du manager de réception à l'épreuve de la digitalisation: cas d'un projet d'accueil en mobilité dans une chaîne hôtelière internationale}

Activity of the front office reception manager in the challenge of the digitalization: case of a project of reception in mobility in an international hotel chain

Pierre Flandrin, Cécile van de Leemput et Catherine Hellemans

\section{NOTE DE L'ÉDITEUR}

Article soumis le 02/02/2021, accepté le 02/09/2021

\section{NOTE DE L'AUTEUR}

Le présent article s'appuie sur des travaux réalisés grâce au soutien financier de Innoviris - projet CAPSMART (2018-Anticipate-025).

\section{Introduction}

1 L'article s'inscrit dans le projet CAP-SMART (2018-2022), visant à identifier le processus d'adoption des technologies intelligentes dans le secteur touristique belge (secteur hôtelier, muséal) et à analyser les impacts sur le travail au sens large (tâche, activité, compétence, emploi). Cette recherche survient dans une période d'incertitude pour les 
acteurs politiques locaux avec la crainte d'une perte d'emplois à faible qualification en raison du développement des technologies intelligentes et de la digitalisation. Une crainte renforcée avec l'apparition en 2019 de la pandémie COVID-19. Une deuxième visée du projet est celle de l'accompagnement par la formation des travailleurs touristiques amenés à intégrer le marché du travail dans un contexte digitalisé. L'étude menée dans ce contexte de recherche s'intéresse plus spécifiquement, à l'activité du manager de réception ${ }^{1}$ dans le cadre de la digitalisation de l'hôtellerie de grande chaîne.

2 Malgré la littérature sur le manager de proximité et les tentatives pour cadrer son travail, la digitalisation a complètement rebattu les cartes de la compréhension de l'activité managériale. Si initialement, le manager de proximité était considéré comme une ressource experte à l'interface entre le «top management» et la «base opérationnelle» (Autissier, \& Vandangeon-Derumez, 2007; Valiorgue, 2016) il est désormais présenté comme un compagnon, un coach, un facilitateur (Ughetto, 2018a) doté de qualités d' "empowerment ${ }^{2}$ » (Benedetto-Meyer, 2019a) ou encore un leader «habilitant» (Caillé, \& Jeoffrion, 2019) permettant aux équipes de travail de se développer et d'être en correspondance avec un milieu professionnel de plus en plus digitalisé (OECD, 2021).

Dans cette perspective, la technologie est souvent décrite dans une visée duale, pouvant tout aussi bien être considérée comme vertu que poison pour le travail managérial. Si cette approche a le mérite d'initier des réflexions prospectives sur les impacts des technologies, elles portent intrinsèquement en elles une vision technodéterministe. Un défaut majeur est qu'elles décomplexifient l'activité des managers, oubliant la part fondamentale faite d'ajustements, de régulations, et de compromis, faisant pourtant l'essence même de cette fonction au cœur de l'organisation (Piney, 2015).

4 La structure de l'article repose sur une analyse des effets de la digitalisation sur l'activité du manager de réception concernant deux niveaux interdépendants. En premier lieu, l'effet de l'environnement digitalisé sur l'activité de travail des managers : les mécanismes à l'œuvre et mis en œuvre par les managers de réception pour inscrire leur activité dans la digitalisation de l'organisation. En second lieu, l'analyse est plus spécifiquement portée sur l'activité des managers de réception dans l'accompagnement d'un projet de transformation digitale complexe et disruptif.

\section{L'Activité comme cadre}

\subsection{L'ergonomie de l'activité comme dépassement des approches duales}

5 Contrairement aux approches duales, l'ergonomie de l'activité et la sociologie de l'activité (Ughetto, 2018b) insistent sur le caractère «situé » de l'activité managériale faite de régulation des situations de travail, parfois critiques, de gestion des variabilités, d'auto-prescription en raison de la faiblesse des prescriptions, de l'incohérence de la multiplicité des règles (Martin, 2013). Si la sociologie met en avant les profondes transformations impactant le travail des managers, elle ne donne pas encore concrètement à voir leurs effets sur l'activité déployée, les nouvelles contraintes et leurs répercussions sur les modes de gestion du collectif de travail et de 
leur santé (Reboul, Delgoulet, Gaudart, \& Sutter, 2020). L'ergonomie considère le manager de proximité comme un travailleur semblable aux autres, pouvant être confronté à des conditions de travail difficiles (Memmi, Rosankis, Sandret, Duprat, Léonard, Morand, \& Tassy, 2020 ; Lamberts, Szekér, Vandekerckhove, Van Gyes, Van Hootegem, Verycken, et al., 2016) car à la frontière d'exigences et de logiques différentes. Des logiques parfois opposées, venues à la fois « du haut» et remontant «du bas» (Daniellou, Boissières, \& Simard, 2010, p. 91). Ce positionnement intermédiaire fait du manager de proximité, une population particulièrement sensible, pouvant être confrontée à des conditions de travail difficiles, parfois dégradantes pour sa santé. Précisément l'étude s'inscrit ici dans le champ de l'ergonomie de l'activité, même si elle s'ancre plus largement dans les fondements et approches en science de gestion, sociologie, psychologie du travail et des organisations. Une activité que nous désignons comme la mobilisation «indissociablement physiologique, cognitive, subjective et sociale» (Coutarel, Caroly, Vézina, \& Daniellou, 2015, p.11). L'inscription dans l'approche ergonomique permet de considérer l'activité avec des propriétés de singularité, émergente de la situation ${ }^{3}$ de travail elle-même inscrite dans un contexte constitué des déterminants "externes" (organisation, système de travail, règles, support collectif, histoire de l'entreprise, histoire du collectif, etc.) et des déterminants «internes » du travailleur (âge, sexe, état interne, compétences, parcours personnel, etc.) de laquelle découle la performance et des effets sur la santé (Grosdemonrouge, 2017, p. 107). Une activité évolutive dans le temps, en fonction des contraintes et astreintes du travail. Deux postulats théoriques orientent cette étude sur une activité touristique peu étudiée jusqu'ici. Premièrement, l'ergonomie fait la distinction entre le «travail prescrit » et le «travail réel » entre lesquels s'exercent les "régulations", éléments centraux de remédiation de ces écarts. Deuxièmement, au niveau conceptuel, l'ergonomie considère les notions de "marges de manœuvre" (Cuny-Guerrier, Caroly, Coutarel, \& AubletCuvelier, 2018 ; Coutarel et al., 2015) et de " pouvoir d'agir »" (Gouédard, \& Rabardel, 2012) « dans » et « sur » la situation, essentielles pour la compréhension des pratiques permises, empêchées et plus largement, permet l'identification du rapport entretenu par le manager de réception avec le collectif médiée par les technologies (Bobillier Chaumon, 2021). À la différence du faire, l'agir suppose que l'action accomplie réponde à des critères de sens : efficacité, authenticité, etc. Autrement dit, le pouvoir d'agir est la capacité de surmonter les obstacles à l'action signifiante grâce à la mobilisation des ressources psychologiques et/ou organisationnelles (Le Bossé, 2003). Le pouvoir d'agir ne se réduit pas à la notion de marge de manœuvre (Clot, \& Simonet, 2015) bien que l'action dépend de l'existence de marges de manœuvre et participe également à en fabriquer. Une articulation de ces notions de pouvoir d'agir et de marge de manœuvre est proposée par Coutarel et al. (2015, p. 18-20). Dans leur conception, le pouvoir d'agir " caractérise de manière générale la relation de l'opérateur à son milieu de travail, audelà des situations singulières et selon une perspective diachronique traversée des histoires collectives» (ibid., p. 18). Le développement du pouvoir d'agir correspond donc à :

«Influencer les processus qui configurent durablement les situations de travail et les marges de manœuvre qui y sont associées. Il s'agit de développer durablement les possibilités (collectives, institutionnelles, temporelles...) de débats de normes, d'explicitation des valeurs qui les sous-tendent et d'arbitrages par ceux que ces débats traversent et affectent. La marge de manœuvre situationnelle s'intègre donc dans un rapport actif au milieu, sur le long terme, construit dans et par les processus de configuration des situations de travail, que nous appellerons le 
pouvoir d'agir. En ce sens, le développement réitéré de marge de manœuvre situationnelle contribue au développement du pouvoir d'agir » (ibid., p. 18).

\subsection{Questionnement de recherche}

6 L'objectif de l'étude est de saisir et d'analyser dans quelles mesures et à quelles conditions, les dispositifs technologiques intégrés dans une logique de digitalisation peuvent être des sources de développement de l'activité du manager de réception. Nous voulons apporter des réponses concrètes sur :

- Comment s'organise cette activité : en dépendance du contexte digital comment les managers de réception structurent leur activité, quelle maîtrise en ont-ils ? Où se situent leurs marges de manœuvre, arrivent-ils à s'en créer pour donner du sens à une nouvelle pratique d'hospitalité et d'accueil médiée par les technologies digitales?

- Comment s'exprime cette activité : à travers quelles formes d'expression, choisies ou contraintes, en proximité ou non, les rôles des managers de réception émergent-ils dans l'activité ?

7 Nous souhaitons également réfléchir à l'accompagnement des transformations digitales par les managers dans le cadre de projets qui touchent directement les pratiques professionnelles d'accueil et plus largement la relation de service. Les questionnements soulevés s'inscrivent dans une temporalité charnière pour l'hôtellerie, avec des grandes chaînes hôtelières qui, à coup de projets digitaux au sein d'hôtels pilotes, poussent les professionnels qui la font vivre à adopter rapidement des comportements d'usage de ces dispositifs. Ces hôtels pilotes constituent des espaces d'expérimentation et de validation, laissant présager un déploiement ultérieur au sein des hôtels de ces chaînes hôtelières.

\section{Digitalisation : une transformation profonde du système touristique}

8 L'introduction des technologies dans la relation de service n'est pas uniquement liée à l'hôtellerie, elle est commune à plusieurs secteurs d'activité: services bancaires, services postaux, transports ferroviaires, grande distribution (Reboul et al., 2020, p. 3). Considérée à large échelle, la digitalisation implique des transformations macroéconomiques qui coöncident avec une refonte des modalités de collaboration avec les clients, les processus de travail internes et parfois même le business model» (Abbasi, Sarker, \& Chiang, 2016). Pour un hôtel, la « digitalisation » ou «transformation digitale" renvoie à l'introduction d'outils numériques dans l'activité de travail remettant en cause de manière profonde les formes du travail, les pratiques professionnelles individuelles et collectives (Benedetto-Meyer, 2019b). On relève de nombreuses tentatives d'adoption des technologies digitales d'un genre nouveau: smartphone, tablette, chatbot, outils de communication instantanée, réseaux sociaux numériques d'entreprise, etc. (Jemine, 2017, 2019). Ces dispositifs promettent une amélioration de l'expérience touristique (Ivanov, \& Webster, 2019) en offrant à chaque client un service personnalisé. Ils promettent également l'enrayement des problématiques touristiques historiquement ancrées et pesantes pour l'employabilité : pénibilité du travail, manque d'attractivité du secteur, turn-over trop important aux 
postes à forte visibilité (Eckert, \& Monchatre, 2009; Forté, \& Monchatre, 2013) identifiés comme la « vitrine de l'entreprise » (Valléry, \& Leduc, 2005).

\subsection{Les évolutions technologiques}

9 L'hôtellerie est un secteur touristique en évolution et nous l'observons dans une période inédite de crise sanitaire (Pillai, Kulshreshtha, \& Korstanje, 2021). Avant cette crise, les dernières décennies ont été marquées par plusieurs évolutions technologiques touchant principalement les procédés de gestion, de communication interne et externe à l'organisation. Les processus de communication avec les Technologies de l'Information et des Communications (TIC) (mail, réseaux sociaux numériques d'entreprise, etc.) ont quant à eux considérablement accéléré la vitesse de transmission des informations. Les logiciels de gestion hôtelière (PMS) et progiciels de gestion ( $\left.\mathrm{PGI}^{5}\right)$ ont rendu accessibles et visibles aux yeux de tous les résultats de l'organisation, les résultats sur la satisfaction des clients, calquant leur modèle de gestion sur celui de la tarification par le "revenue management $»^{6}$ (Denizci Guillet, \& Chu, 2021). Cette pratique s'est renforcée à la suite du développement des technologies de l'information et prévoit de se renforcer encore avec l'usage des systèmes d'intelligence artificielle dans les entreprises de service (Gamkrelidze, Barcellini, \& Zouinar, 2021).

\subsection{La révolution de I'Intelligence Artificielle}

Parce qu'elle se voit alimentée par la massification des données et l'amélioration de la puissance de calcul, la révolution de l'Intelligence Artificielle vient couronner cette évolution fournissant des informations essentielles sur les clients (avec les dispositifs de gestion de la relation client $^{7}$ ), et les tâches réalisées par les collaborateurs. Évolutions successives et révolution de l'IA permettent aujourd'hui de combiner une pluralité de dispositifs technologiques, intégrés à large échelle dans les organisations, visant à les rendre plus agiles et flexibles à la demande.

11 La flexibilité des travailleurs est considérée comme un facteur de survie avec le développement de nouvelles formes de contractualisation basées sur la stratégie de l'«asset light $»^{8}$ (Grefe, \& Peyrat-Guillard, 2019) et la flexibilisation du marché touristique imposée par certains acteurs numériques. C'est le cas de l'arrivée des plateformes telles que "Airbnb ", qui ont un effet disruptif sur la chaîne de valeur touristique, ou encore, des agences de voyages en ligne ("Booking.com», "Expédia» "Kayak.com", "Hotels.com» "Travelocity", etc.) qui d'une certaine façon accentue le culte de l'urgence et de l'immédiateté (Felio, 2014) par la mise en relation des structures d'hébergement et les clients. Devant la concurrence imposée par les plateformes numériques, les chaînes hôtelières se sont vues " obligées » d'innover. Les effets émergents ont été notamment : (i) l'extension de la relation de service, hors du strict cadre physique de l'hôtel, renforçant les possibilités d'actions des clients et leurs exigences vis-à-vis des hôteliers; (ii) de s'orienter vers des pratiques d'accueil innovantes et la proposition de nouveaux services pour fidéliser les clients. Pour les travailleurs de l'hôtellerie, derrière ces innovations, et la récurrence des changements, il faut surtout y voir des conditions d'exercice remodelées (Bolduc, \& Baril-Gingras, 2010) et avec elles, une transformation de la relation de service. 


\section{L'adoption des technologies digitales par les organisations hôtelières : une vision controversée sur les rôles et le travail managérial}

\subsection{Le manager de réception une fonction charnière dans l'hôtellerie}

Premier échelon hiérarchique au cœur de l'organisation hôtelière, le manager de proximité de la réception, plus communément appelé front office manager fait office de fonction tampon entre l'équipe de réceptionnistes et les clients-touristes (QuératHément, 2014). Il se présente avec les réceptionnistes comme les figures humaines, points de repère de la qualité perçue et de la satisfaction des clients. Au niveau de l'organisation, le manager est le garant de la performance opérationnelle de la réception. C'est sur lui, que repose la mise en place, le respect et l'évaluation de la bonne mise en application des règles, des procédures définies par l'organisation. Le manager de réception évolue aujourd'hui dans des conditions de travail spécifiques accomplissant des tâches multiples et variées, techniques, relationnelles et commerciales, relatives à la gestion des ressources humaines et à la production. Aujourd'hui, ces tâches tendent à s'élargir, en particulier en matière de communication, d'utilisation de nouvelles technologies et de gestion, d'exigences de gestion et de contrôle, et les attentes à leur égard décrites en termes de développement de «nouvelles compétences » sont grandissantes (Wolff, 2013).

\subsection{Des études centrées sur les « rôles » des managers de proximité}

Contrairement à la vision fonctionnaliste développée par Fayol en 1916, les conceptions modernes considèrent le travail des MDP comme une activité émergente. Les études et analyses menées dans le courant du «content managerial work» (Lanoë, 2019) ne manquent pas pour décrire le travail des managers. Il faut remonter à Mintzberg (1973) pour retrouver une classification des rôles managériaux en trois catégories : les rôles interpersonnels reprenant les fonctions de figure de proue, de leader et d'agent de liaison, les rôles liés à l'information faisant référence à la collecte et à la distribution des informations ainsi qu'à la fonction de porte-parole et enfin les rôles décisionnels tels que l'entrepreneuriat, la gestion des conflits, la négociation et l'allocation des ressources ». Plus récemment, Guilmot et Vas (2012) ont proposé un cadre conceptuel qui articule les différents rôles des managers de proximité. Autant de rôles identifiés qui, malgré certaines nuances, se rejoignent pour qualifier une fonction d'apparence polyvalente et multiforme. Fort de ces postulats, les études sur les rôles ont donné lieu à l'élaboration de différentes grilles de lecture pour donner à voir le travail des MDP. Devrait-on dire avec plus de précisions, « les » travail des MDP. Ces « travail » relèvent de diverses formes de travail: le travail d'«organisation" (Dujarier, 2006), d'« encadrement» (de Terssac, \& Cambon, 1998), d'« articulation » (Dehame-Leleyter, Lancry, \& Vallery, 2008 ; Grosjean, \& Lacoste, 1999) de «traduction » (Desmarais, \& Abord de Chatillon, 2010) ou encore d'«arrangement", de "conformation», d'«enrôlement" pour venir caractériser l'activité des MDP. Chaque travail y est spécifié à travers l'analyse située de l'activité dans la situation et le déroulement de l'action. Une fois ces éléments pris en considération, les rôles émergent alors sous une 
variété de formes expressives dans l'action (" animation ", « contrôle ", « supervision ", " coordination", " pilotage" "formation " "régulation », etc.). Dans le domaine du management $\mathrm{du}$ changement, trois thèmes restent privilégiés: les outils qui permettent de bien manager le changement, le rôle du leader visionnaire et le vécu de la base opérationnelle (Oiry, \& Vignal, 2016). Plusieurs travaux ont également mis en perspective le rôle des managers dans l'accompagnement des changements organisationnels (Guilmot, \& Vas, 2012) à travers un rôle dans la construction du sens (Weick, 1993) exercé vers les membres de son équipe sur la pertinence du changement proposé (Autissier, Johnson, \& Moutot, 2015). Rouleau et Balogun (2007) ont ainsi montré les mécanismes concrets de sensemaking et de sensegiving par lesquels cette construction du sens se joue. En revanche, ils n'ont pas explicitement pris en compte le fait que les managers de proximité sont dans une situation singulière, ils vivent le changement et en même temps, ils doivent le déployer et le défendre devant leur équipe. Weick (1993) insiste pourtant sur le fait que le changement remet en cause la répartition des tâches, des responsabilités et de l'autorité et nécessite pour les individus de reconstruire mentalement un système de rôles adapté à la situation (Autissier, Vandangeon-Derumez, \& Vas, 2010).

Si aucune menace ne semble planer autour de la figure du manager de réception contrairement à d'autres secteurs où le management de proximité est pour partie algorithmique (Galiere, 2020, 2021; Lee, Kusbit, Metsky, \& Dabbish, 2015), les recherches font entrevoir des reconfigurations de son activité et des rôles managériaux.

\subsection{Désincarnation du travail des managers de proximité avec la digitalisation}

15 En référence aux différentes évolutions se dégage une première vision du MDP à la fois hyperconnecté technologiquement (Prost, \& Zouinar, 2015), déconnecté, désincarné et mis à distance du terrain par une multitude de tâches à gérer (Dujarier, 2017). Les écrits de Detchessahar (2011) dénoncent notamment les conséquences d'un management empêché des encadrants de proximité, déconnectés de leurs subordonnés, trop occupés à alimenter les outils de gestion ou trop accaparés par tout un ensemble de groupes de travail découlant du développement du management participatif. L'activité de "gouvernant" est dans l'hôtellerie un exemple signifiant du virage gestionnaire. Équivalent du manager de réception pour le service de nettoyage, les gouvernants sont désormais dotés d'algorithmes pour le contrôle du travail du personnel de nettoyage. Dans un secteur largement sous-traité aux impacts bien démontrés sur la dilution des collectifs de travail (Lizé, 2020), les applications sur smartphone s'imposent désormais comme de nouveaux supports managériaux permettant de garder le contrôle sur l'activité finalisée ou directement en train de se réaliser. L'obligation pour les travailleurs du nettoyage de renseigner leur activité à travers une standardisation de la remontée d'informations sous forme de codes préétablis, semble affecter leur activité régulièrement décrite en termes de «digital taylorism » (Brown, Ashton, \& Lauder, 2010) ou de «datafication» du travail (Stein, Wagner, Tierney, Newell, \& Galliers, 2019). Supposés être plus performants et plus efficaces pour certaines tâches les systèmes technologiques pourraient aller jusqu'à substituer le manager de proximité dans l'accomplissement de certaines d'entre-elles (Gobble, 2013; Lee et al., 2015) pour lui permettre d'en réaliser d'autres avec plus de valeur ajoutée. Une tendance qui serait 
plus marquée dans les grands groupes où de nombreux projets de transformation, le plus souvent digitaux, viennent se superposer. Le mal-être des MDP dans ces organisations y serait en partie caractérisé par de la sur-connexion technologique, une grande fragmentation de l'activité de travail (Bobillier Chaumon, Sarnin, Cuvillier, \& Vacherand-Revel, 2015), un manque de moyens, de temps, et des conditions de travail insatisfaites pour couvrir avec efficience l'ensemble des tâches à réaliser. Une fonction en l'occurrence aux contours mal définis pouvant déborder sur les autres sphères de vie (Hellemans, \& Vayre, 2021). Ce débordement serait favorisé par une pression technologique forte qui véhicule l'existence d'un «risque techno social» duquel découleraient des tentatives pour distancier les managers de la technologie, au moyen de mesures imposées, comme la « déconnexion volontaire » (Jauréguiberry, 2013; Oliveri, \& Pélissier, 2019).

\subsection{Vertus émancipatrices des technologies digitales pour le travail managérial}

16 Parallèlement à cette vision d'hyper-connexion désincarnée, une autre vision affirme que les technologies digitales émergentes sont à considérer comme une avancée pour le travail des MDP. La perspective des auteurs portant cette vision techno-déterministe est celle d'un MDP qui se verrait libéré, réincarné (Dujarier, 2017) dans une relation de proximité avec son équipe (Piney, 2015). Une relation de proximité qui ne se limiterait pas à la seule dimension physique, mais qui pourrait prendre en compte la proximité technique et socioculturelle, celle où managers et collaborateurs peuvent partager le même univers professionnel, un langage, une même conception du métier, voire des valeurs (Srajek, 2003). Largement décrite par Piney (2015), cette relation relève d'un jeu d'équilibre de la proximité dans le cours même de l'activité de travail. Dans les organisations digitalisées, l'exercice de la proximité pourrait dès lors fortement dépendre des marges de manœuvre pour supporter et faciliter les pratiques des managers, notamment en matière de monitoring des équipes, de gestion des risques, de prise de décisions, de partage d'informations et de coordination du travail. Le " management algorithmique " par les "systèmes intelligents » permettrait aussi de superviser des travailleurs à large échelle mieux que ne le ferait le management par des hommes, avec plus d'équité, mais aussi de piloter au lieu de l'aider à la prise de décision dans les organisations (Newell, \& Marabelli, 2015). En termes d'effets, ces changements pourraient induire de nouvelles expressions de rôles du management, davantage en proximité avec les collaborateurs. Le management serait le grand gagnant de ces transformations technologiques (Barton, \& Court, 2012). Les rôles comme la supervision et le contrôle pourraient par exemple être pris en charge par les technologies digitales, offrant des marges de manœuvre nouvelles, donnant une liberté d'exercice aux managers.

17 Les effets de ces technologies sur le travail sont vus comme un potentiel enfermement ou au contraire une ouverture de l'activité managériale vers de nouveaux champs d'action et de connaissances, des expériences de travail nouvelles requérant des habiletés cognitives, comportementales et techniques (Bobillier Chaumon, 2021). Ces technologies suscitent aussi d'autres formes de collaboration à concevoir et de mise en réseau des équipes que nous avons approchées par la démarche suivante. 


\section{Construction de la démarche de recueil des données}

\subsection{Une étude de cas inscrite dans un projet de recherche plus global}

L'étude de cas multiple ${ }^{9}$ (Guerrier, 2016 ; Yin, 2009, p. 46) fait partie des recherches du projet CAP-SMART «Développement des Capacités pour le Suivi et la Maîtrise de l'Adoption de la Robotique et autres technologies intelligentes dans le Tourisme bruxellois» (octobre 2018-octobre 2022). Ce projet est financé par l'organisme bruxellois Innoviris et rassemble des partenaires universitaires interdisciplinaires (i) un laboratoire de recherche orienté en psychologie du travail et ergonomie et (ii) un laboratoire spécialisé en tourisme. Des acteurs territoriaux et des organismes professionnels (Brussels Hôtel Association, Conseil bruxellois des Musées, Horeca formation, Visit.Brussels) constituent des ressources sur lesquelles ont pu s'appuyer les chercheurs pour identifier des structures hôtelières cibles. Les attentes de ce projet sont liées aux préoccupations du devenir des travailleurs des organisations touristiques bruxelloises étant donné le développement des technologies intelligentes (TI), mais il est également centré sur la compréhension du processus d'adoption des TI à travers une approche systémique mêlant le niveau micro, méso et macro du système touristique (Camus, Hikkerova, \& Sahut, 2010; Yatchinovsky, 2018). Les données récoltées relèvent d'un processus de construction sociale basé sur la confiance est entretenu d'octobre 2019 à avril 2021 avec trois hôtels d'une même chaîne multinationale « $\mathrm{GRZ}^{10}$ ». L'étude de cas ne dépend pas d'une relation conventionnelle établie entre les chercheurs et la chaîne hôtelière.

\subsection{Contexte du terrain de recherche}

Le plan stratégique de digitalisation lancé en 2014 par la chaîne d'hôtels GRZ montre la place de choix accordée à la mise en place de la digitalisation dans le fonctionnement du groupe hôtelier. La chaîne hôtelière a lancé le plan « Leading Digital Hospitality » dont une des pièces maîtresses est " la montée en puissance du mobile, la personnalisation des services et [la] fluidité du parcours des clients avant, pendant et après leur séjour ». Les structures hôtelières retenues dans l'étude, appartenant ou liée à $G R Z$, l'ont été sur la base de 3 critères principaux: le standing, le type d'organisation (franchise ou contrat de gestion) et l'intégration dans une démarche de transformation digitale (Tableau 1). Deux hôtels managés de standing 3 et 4 étoiles et 1 hôtel franchisé de standing 3 étoiles ont été retenus. Ils participaient tous en tant qu'établissements pilotes, à l'expérimentation du même dispositif mobile de réception qui sera nommé ici Hospitality mobile. 
Tableau 1 : Caractéristiques principales des hôtels pilotes et de la population. Table 1: Description of test Hotels and population

\begin{tabular}{|c|c|c|c|}
\hline & Hôtel A & Hôtel B & Hôtel C \\
\hline \multicolumn{4}{|c|}{ Caractéristiques générales des hôtels } \\
\hline Standing & 3 étoiles & 4 étoiles & 3 étoiles \\
\hline Chambre & 184 & 140 & 225 \\
\hline Taux d'occupation annuel \% & $90 \%$ & $95 \%$ & $90 \%$ \\
\hline \multicolumn{4}{|l|}{ Spécificité des hôtels } \\
\hline Mode de gestion & Franchise & $\begin{array}{l}\text { Contrat de } \\
\text { management }\end{array}$ & Contrat de management \\
\hline Localisation & Centre-ville & Centre-ville & Proximité de la gare \\
\hline $\begin{array}{l}\text { Nombre de réceptionniste par } \\
\text { poste }\end{array}$ & 2 & 2 & 2 \\
\hline Sécurité & Moyenne & Optimale & Sensible $24 \mathrm{~h} / 24$ \\
\hline Cadence & Haute & Moyenne & Haute \\
\hline $\begin{array}{l}\text { Clientèle semaine/ } \\
\text { Clientèle week end }\end{array}$ & $\begin{array}{l}\text { Business } \\
\text { Famille } \\
\end{array}$ & $\begin{array}{l}\text { Business } \\
\text { Famille }\end{array}$ & $\begin{array}{l}\text { Business } \\
\text { Famille } \\
\end{array}$ \\
\hline \multicolumn{4}{|c|}{ Caractéristiques des managers de réception } \\
\hline Âge moyen (genre) & 32 (Femme) & 27 (Homme) & 32 (Homme) \\
\hline $\begin{array}{l}\text { Ancienneté sur le poste } \\
\text { (ancienneté du métier) }\end{array}$ & $\begin{array}{l}2 \text { ans } 4 \text { mois } \\
(10 \text { ans })\end{array}$ & $\begin{array}{l}2 \text { ans } 4 \text { mois } \\
(4 \text { ans et } 5 \text { mois })\end{array}$ & $\begin{array}{l}2 \text { ans } 6 \text { mois } \\
(2 \text { ans et } 6 \text { mois })\end{array}$ \\
\hline
\end{tabular}

\subsection{Méthodes de collecte des données}

20 Les méthodes mobilisées pour le recueil des données ont été chronologiquement les suivantes: des entretiens exploratoires (a) et d'approfondissement (b), des observations des activités de réception hôtelière (c), des suivis d'activité des MDP (d), associés à une verbalisation simultanée (Leplat \& Hoc, 1983) et des entretiens complémentaires (e). Nous présentons l'objectif du recours à chaque méthode dans les sous parties ci-dessous.

\subsubsection{Entretiens exploratoires (a) et d'approfondissement (b)}

En première démarche, des prises de contact avec des hôteliers de la région de Bruxelles Capitale ont permis la réalisation d'entretiens exploratoires auprès de divers acteurs clés, dont l'objectif visait la compréhension du regard porté vis-à-vis de la digitalisation et l'étude des conséquences potentielles sur l'activité de travail. Les professionnels rencontrés étaient des MDP ainsi que d'autres acteurs de l'hôtellerie: réceptionnistes, dirigeants d'hôtel, directeur des applications IT d'un grand groupe hôtelier. Suite à la phase des entretiens exploratoires, des entretiens d'approfondissement ou entretiens semi-directifs contextuels ont ciblé l'activité des managers de réception. Un guide d'entretien a été conçu à cet effet regroupant quatre thèmes: (1) l'identification de la nature de l'activité (tâches, projets, outils utilisés, interlocuteurs...), (2) l'ancrage et l'identification des ressources individuelles et collectives nécessaires à la réalisation du travail et notamment la détermination du contexte socio-organisationnel dans lequel l'activité s'inscrit (procédures, normes, standards, prescrit, structure hiérarchique...), (3) la place des technologies dans le réel de l'activité, et (4) les apports, les freins et les attentes quant à la technologie. Ces entretiens ont été réalisés auprès de travailleurs (manager de réception, réceptionniste) du groupe hôtelier GRZ pleinement engagé dans un processus de transformation digitale. 


\subsubsection{Observations générales de la réception (c) et suivis d'activité des managers de réception $(\mathrm{d})$}

L'étude de cas a donné lieu à des observations des services de réception dans les trois hôtels A, B, C sur 3 demi-journées de haute occupation, avec un important nombre de départs et d'arrivées de clients. Les objectifs visés étaient: la compréhension des relations et interactions des réceptionnistes avec le MDP, le fonctionnement général du service avec prise en compte des dimensions collectives et collaboratives du travail à la réception. Nous avons notamment réalisé dans ces trois demi-journées une analyse vidéo permettant de relever les déplacements proactifs des réceptionnistes vers les clients ainsi que le partage d'attention entre le dispositif et le client. Pour comprendre l'activité des managers de réception, ces derniers ont fait l'objet d'un suivi d'activité individuel sur une journée à forte activité, caractérisée par un taux d'occupation supérieur à $95 \%$. La compréhension située du travail des MDP reposait sur une théorie ancrée " grounded theory ( (Glasser \& Strauss, 2010). Une théorie inductive visant à faire émerger les rôles des MDP à partir de leurs activités sans l'élaboration d'hypothèse formulée à priori.

\subsubsection{Entretiens complémentaires (e)}

Afin de constituer une vue holistique, des entretiens complémentaires ont été réalisés pour élargir notre regard sur le fonctionnement global des hôtels du groupe, sur la stratégie de digitalisation de GRZ et sur les relations sociales au sein du groupe hôtelier. Les acteurs concernés par ces entretiens étaient le directeur IT, un représentant syndical également réceptionniste, un manager général d'hôtel, un revenue manager senior commun aux trois hôtels retenus.

\subsection{Méthodes d'analyse des données}

Après retranscription, les entretiens exploratoires et d'approfondissement ont été analysés par la méthode d'analyse de contenu thématique (Bardin, 2013). Cette méthode consiste à repérer parmi des expressions verbales ou textuelles des thèmes généraux récurrents qui apparaissaient sous divers contenus plus concrets et à les analyser selon un codage mixte combinant des thèmes établis $a$ priori et des thèmes qui ont émergé de l'analyse des données. L'analyse des observations et du suivi d'activité des MDP a été réalisée sur base de la méthode de l'analyse ergonomique de l'activité (Leplat, 2015). Pour les observations, le chercheur a utilisé la méthode du papier crayon. Le suivi d'activité se présentait, sous la forme d'une chronique d'activité dans laquelle le chercheur accompagnait chaque MDP pendant une journée de travail en prenant en note, avec des indications temporelles, l'ensemble des activités réalisées par ce dernier, ses interactions avec le service de réception, les autres services, les clients, etc., en direct ou au téléphone. Des verbalisations ont été recueillies quand la situation le permettait. Dans le cas contraire, le retour sur l'activité se faisait en post-activité. Les verbalisations ont été enregistrées, permettant d'inférer à la fois la contrainte cognitive, les représentations et les vécus des MDP dans le déroulement de l'action. L'activité recueillie a ensuite été découpée en séquences d'activité pour distinguer l'expression de différents rôles (régulateur, animateur, superviseur, etc.) (Tableau 2). 
Tableau 2 : Découpage de l'activité en séquence codée en " expression de rôle ". Table 2: Splitting the activity into sequences coded as "rôle expression"

\begin{tabular}{|l|l|}
\hline \multicolumn{1}{|c|}{ Séquence d'activité } & \multicolumn{1}{|c|}{ Expression des rôles } \\
\hline $\begin{array}{l}\text { S'occupe de la relation client d'un client « Gold » à la suite } \\
\text { d'un sous classement pour cause d'overbooking }\end{array}$ & Régulateur (erreur) \\
\hline Traite le cas vidéo de la scène d'un vol récent & Régulateur (problème) \\
\hline Prépare les évaluations individuelles du personnel & Évaluateur \\
\hline $\begin{array}{l}\text { Présente collectivement différents projets de } \\
\text { responsabilisation à chaque réceptionniste et anime sur } \\
\text { tableau blanc la situation }\end{array}$ & Coordinateur \\
\hline $\begin{array}{l}\text { Vérifie par téléphone les no-show auprès des } \\
\text { réceptionnistes }\end{array}$ & Superviseur/contrôleur \\
\hline
\end{tabular}

\section{Résultats}

La présentation des résultats en deux parties donne d'abord à voir l'environnement digitalisé de travail de l'activité quotidienne des managers, puis l'activité des managers dans le cadre de l'adoption du projet Hospitality mobile.

\subsection{L'activité du manager de réception dans l'environnement digitalisé de $G R Z$}

C'est à partir des entretiens exploratoires, d'approfondissement et des suivis d'activités que ces analyses ont été réalisées afin de développer une compréhension de l'activité des MDP de la réception dans un environnement digitalisé. Nous présentons dans les sous-parties suivantes des éléments principalement relatifs à la chaîne GRZ, que l'on retrouve également dans nos autres entretiens.

\subsubsection{Une activité cadrée par les outils de gestion, renforcée avec la digitalisation}

Premièrement, les observations confirment que l'activité des managers de réception est en proximité physique avec l'équipe de réception. Le poste est ainsi marqué par la constante perméabilité du poste de travail aux collaborateurs (personnel de la technique, de la réception, les femmes de ménage, le dirigeant) et le maintien à distance de son attention sur l'activité des réceptionnistes par les écrans de vidéosurveillance (Figure 2). 
Figure 1 : Poste de travail du manager de réception (Hôtel B) Figure 1: Front desk manager's workstation (Hotel B)

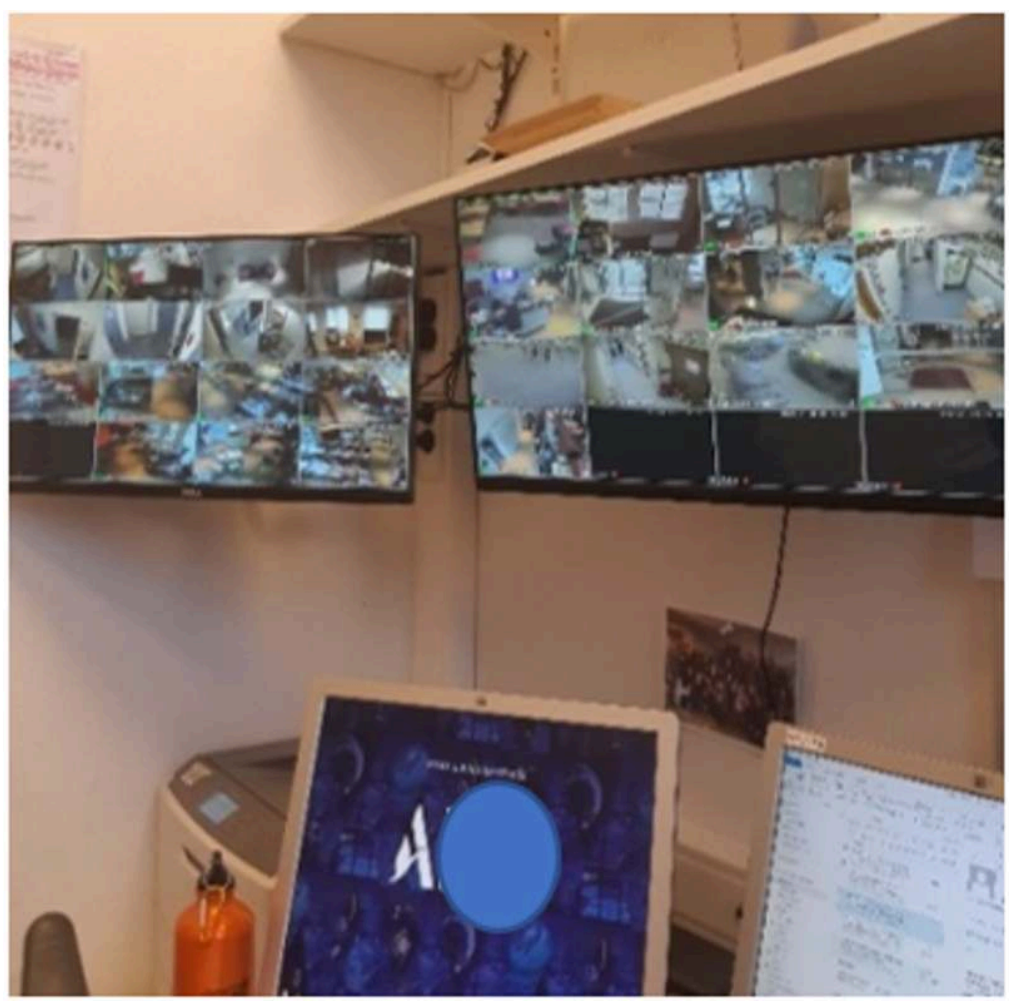

L'activité des managers de réception s'apprécie dès la prise de poste au gré des connexions sur les logiciels de gestion qu'ils utilisent quotidiennement et de ceux utilisés ponctuellement pour gérer les variabilités des situations qu'ils rencontrent. Sur 394 outils disponibles dénombrés, les managers jonglent avec une dizaine de dispositifs de gestion selon les besoins de la situation à gérer. Le champ d'application recouvre des domaines tels que la gestion de la satisfaction, la gestion des réservations, la gestion de la relation avec les sociétés, la gestion des ressources humaines.

«Je gère la gestion des réservations, la gestion des arrivées, les mails, les questions qui viennent en plus, la gestion des conflits inter-département mais aussi les clients un peu difficiles, la gestion des commentaires » (MDP, extrait d'entretien $n^{\circ} 1$ ).

Une unique situation à gérer peut par ailleurs, obliger les managers à se confronter à une multiplicité d'écrans et de logiciels de gestion pour visualiser les données et comprendre la situation d'un seul client. C'est le cas de la situation d'overbooking ${ }^{11}$ mal gérée par l'équipe de réception de nuit au sein de l'hôtel B qui avait donné lieu au déclassement d'un client fidèle vers l'hôtel $C$, hôtel de plus bas standing. Le manager de réception tente alors de réparer à «chaud» la situation, en trouvant le plus rapidement possible le moyen de s'excuser auprès de ce client, devant la menace potentielle d'une évaluation négative sur la plateforme digitale d'e-réputation («voice of the guest ») centralisant l'ensemble des avis clients.

Le constat dressé par ces managers à travers cette situation parmi d'autres est que la digitalisation, avec l'intégration massive des données clients intensifie leur activité et amène à ce que nous qualifions, une " gestionnarisation » de la relation de service, si bien que chaque situation est elle-même orientée vers une gestion particulière. Plus pressante et surtout plus pesante, l'implication des indicateurs de gestion alimentés par le Big Data est traitée dans des outils de gestion internes (progiciel de gestion, 
logiciel de gestion de la relation client) devenus le support du travail des managers. Les managers disposent d'indicateurs de résultats internes en termes de revenus: RevPar («Revenue per Available Room»), d'indicateurs de suivi : RPS ("reputation performance score »), d'indicateurs de reporting. Le travail consiste à se rapprocher au plus près des prescriptions de la hiérarchie, fortement impulsées par les planneurs ${ }^{12}$ (Dujarier, 2015) et les pilotes ${ }^{13}$ (" revenue managers») qui orientent sur la stratégie à suivre.

«On a des revenus managers qui s'occupent de fixer les prix et la stratégie. Pour avoir [...] une vision de comment on va évoluer, c'est ça qu'ils nous donnent. Pour le mois de janvier ce qui a été forecasté, ce qui a été prévu, on doit se retrouver avec ces chiffres-là, le taux d'occupation à $85 \%$, un prix moyen de $133 €$, un revenu de $500000 €$, un revpar (revenu par chambre) de $114 €[$ [...]. Je fais en sorte de faire un maximum de revenu. » (MDP, extrait d'entretien $\mathrm{n}^{\circ} 2$ ).

31 Sans directement être des supérieurs hiérarchiques, ces pilotes présentent un arsenal algorithmique capable d'orienter le plus souvent à distance les conduites des MDP. C'est principalement le cas des revenue managers, par l'intermédiaire desquels les MDP des hôtels A B C, reçoivent chaque jour des données automatiquement prélevées sur la tarification de dix hôtels, sélectionnées en amont pour leurs caractéristiques similaires.

«Sur certains hôtels de Bruxelles maintenant, c'est la machine qui va faire le travail et qui va lui-même mettre les stratégies d'ouverture, de fermeture, de pricing, etc. Notre système est un algorithme intelligent, une intelligence artificielle qui donne déjà des recommandations, mais pas totalement automatisé » (Revenue Manager Senior des hôtels A B C, extrait d'entretien $\mathrm{n}^{\circ} 3$ ).

Le mode de pilotage régi par la donnée renforce l'expression du pilotage de la performance avec une orientation marquée vers la maximisation du revenu et du chiffre d'affaires de l'hôtel. Les MDP sans y être explicitement «obligés » tentent de respecter la marche à suivre qui est de se conformer aux objectifs chiffrés qu'on leur donne. Les managers doivent aussi rendre compte dans le cas d'un rejet d'overbooking proposé par les pilotes, des raisons de ce choix, endossant ainsi à eux seuls toute la responsabilité d'une éventuelle perte de revenu.

\subsubsection{Le pilotage de l'activité managériale par les revenue managers}

Pour les managers de réception, la « chambre » en tant qu'unité marchande est l'objet de toutes les attentions quotidiennes, observée et suivie à travers les dispositifs technologiques de gestion interne. L'objectif consiste pour ces managers connectés à valoriser au plus "vite» chaque référence de chambre sur les plateformes de réservation en ligne (Booking.com, Expédia, Tripadvisor...).

« Çà c'est le tableau des stocks. Je check tout le temps parce que c'est les ventes. Le DBB c'est le double standard. Puisque c'est le $1^{\text {er }}$ prix visible sur Internet, c'est les chambres qui partent le plus vite. En fait on fake le système, on fait comme si on vend 30 chambres de plus en DBB [...]. Mais le jour même on va les réattribuer dans d'autres types de chambre, des gens vont avoir des sur-classements gratuitement. [...]. La stratégie au niveau du revenu c'est que plus tu vends de chambres plus ton prix va augmenter la différence de prix entre une DBB et un autre type de chambre famille ce n'est pas grand-chose. On ne va pas faire d'argent si on fait plus de DBB mais on va faire plus d'argent si on vend plus vite des DBB. Donc on est plus vite en restreint, pas beaucoup de disponibilités, et là on peut monter les prix et c'est là que l'on va gagner de l'argent » (MDP hôtel B, extrait de verbalisation, $n^{\circ} 4$ ).

Les recommandations formulées par les pilotes, proches de l'injonction sont difficilement tenables et nécessitent une supervision constante des informations 
numériques sur le cloud. Un suivi constant de l'évolution en fonction des différents canaux de réservation sollicités par les clients. Cette activité de supervision révèle des choix dans l'anticipation des comportements de réservation des clients, fondés sur des calculs complexes, intériorisés qui amènent les managers dans des stratégies d'action et de prise de décision non visibles par la direction du groupe hôtelier. Ils agissent et orientent leur activité en faisant ainsi référence au « revenu moyen par chambre ». En agissant de la sorte, les managers parviennent à s'écarter du cadre prescrit afin de préserver les équipes en évitant des tâches coûteuses et inutiles.

«Si on peut mettre quelqu'un qui a réservé tout seul dans une DBB dans une salle

famille parfait !! Finalement, toutes les chambres sont vendues, donc pas beaucoup

de coût [...] moins de travail pour le service housekeeping et la réception. Ils (les

réceptionnistes) doivent toujours regarder si on a des disponibilités en DBB ».

(MDP hôtel B, extrait de verbalisation $\mathrm{n}^{\circ}$ 5)

Les choix fondés sur leur expérience impactent directement malgré eux les réceptionnistes qui se retrouvent eux aussi embarqués dans des choix de gestion, quand ils doivent par exemple expliquer à des clients en recherche d'une chambre qu'il n'y en a «fictivement» plus pour 1 nuit, mais qu'elle est disponible en cas de réservation de minimum 2 nuits. Pour un MDP, rendre lisible sa stratégie aux acteurs de la réception qui ne comprennent pas toujours les raisons de laisser par exemple «temporairement une chambre disponible, en indisponibilité » devient une compétence appréciée par les réceptionnistes. La lisibilité de la stratégie pour les acteurs de terrain ne se limite pas au service de réception, elle s'étend aux pilotes qui en sont éloignés. Ces tâches de reporting supplémentaires que les managers s'imposent, et qui viennent s'ajouter au reporting quotidien, sont jugées vitales pour donner un aperçu aux pilotes de la vie de l'hôtel (travaux en cours, etc.), mais surtout capitales pour générer des marges de manœuvre collectives futures. Les managers sont en effet conscients que la recommandation par l'IA du revenu à atteindre l'année suivante se base essentiellement sur les résultats de l'année écoulée.

\subsubsection{Une digitalisation mettant à l'épreuve l'activité managériale, un positionnement à construire : entre contrôle et régulation du travail des réceptionnistes}

Un pouvoir de contrôle renforcé par la digitalisation, mais peu exploité...

36 Si l'ambition des MDP est de rendre compte de la vie de l'hôtel et de "l'activité réelle » s'y déployant, on constate la difficulté à le rendre visible par les outils existants qui se fondent essentiellement sur des données quantifiées. Contrôlés de part en part, les MDP en deviennent eux-mêmes contrôlant pour leurs équipes.

«Je regarde tout ce qu'ils (les réceptionnistes) réduisent, les remboursements, les réductions, les trucs qu'eux font en réception. Par exemple, ils font un cadeau à un client [...] Tout ce qui est no show (les clients qui ne se présentent pas), s'ils vont venir après. Et puis il y a [...] des réservations en last-minute non remboursables. Tous les séjours gratuits il faut savoir pourquoi. Tout ça, je contrôle si c'est bien chargé ». (MDP, extrait de verbalisation $\mathrm{n}^{\circ}{ }^{6}$ ).

Un contrôle facilité avec la digitalisation qui permet la parcellisation des processus de travail. L'accueil réalisé sur smartphone, tablette ou ordinateur est fragmenté en micro-tâches codifiant l'activité des réceptionnistes et grâce auxquelles des indicateurs 
de suivi de la performance sont calculés automatiquement (durée de réalisation du check-in, nombre de sur-classement par client, etc.).

«Avant que le programme (PMS de l'entreprise) ait été mis sur place, si un travailleur il n'avait pas fait un upgrade à un client ou il n'avait pas fait les choses de manière correcte, il avait toujours l'avantage que le manager n'allait jamais le savoir. Il y a un contrôle derrière parce que vous êtes obligés de faire un check-in complet. Ce qui force les travailleurs à offrir certains services avant de finaliser les check-in. Donc ça fait un contrôle beaucoup plus exhaustif. Par exemple, la première étape, ça va être que le client il doit montrer sa carte d'identité, puis donner une méthode de paiement. Le réceptionniste doit offrir la possibilité de faire un upgrade donc un sur classement dans notre chambre supérieure. Et pour pouvoir passer cette étape, Il doit cliquer (sur son smartphone) que le client ne veut pas ce service. Et donc c'est enregistré que le travailleur a cliqué non... le pourcentage d'upgrade qui se passe le lundi, mardi... Donc tout ce type de data c'est très important pour pouvoir faire plus d'argent. Et en fait c'est ce qu'on est en train de faire » (extrait d'entretien $\mathrm{n}^{\circ}$ 7)

Cette activité de contrôle est d'autant plus importante que l'atteinte des objectifs chiffrés engendre une reconnaissance individuelle, associée à l'attribution de bonus.

«En même temps, moi j'ai mes objectifs mes bonus à moi et je cale tous les objectifs de mon équipe là-dessus aussi, j'essaye de prendre la compétence de chacun pour arriver à mes fins ». (MDP, extrait de verbalisation $n^{\circ} 8$ ).

\section{Une activité de régulation prégnante et essentielle}

Faire "rouler" ou plutôt "cadrer" le travail ne se résume pas simplement à se conformer au cadre prescrit et à contrôler le travail des réceptionnistes. C'est surtout faire en sorte que les imprévus et les aléas ne viennent pas perturber les prescriptions, lorsque les règles et les procédures sont floues. C'est le cas, lorsqu'une énième fois, le MDP est interrompu dans sa tâche par « un client fidèle et régulier [qui] prévient de son départ prématuré 3 jours avant » lui laissant un temps précieux pour réattribuer sa chambre. Le MDP réunit et consulte alors le collectif de réception autour d'une formulation de remerciement, en se basant sur la base de données clients indiquant préférences et goûts de ce dernier permettant au réceptionniste de sortir de l'hôtel pour ramener le parfum apprécié de ce client. La chronique reprise en séquence d'activités sur 30 minutes (Tableau 2) est une des illustrations de cette activité de régulation et de contrôle préalablement développée. Si la digitalisation offre aux MDP un pouvoir de contrôle plus important, les usages qu'ils en font relèvent plutôt de la régulation et de la gestion du service dans l'optique de maintenir une qualité de service acceptable et équivalente aux yeux de chaque client.

«Pour le chatbot, ce qu'on a remarqué c'est qu'en semaine par exemple... on arrivait toujours à répondre assez rapidement parce que moi [manager de réception] je ne travaille pas le week-end par exemple et dans les bureaux derrière tu as une personne qui va s'occuper aussi de tout ce qui est community et de clients et donc en semaine donc moi je répondais, ou elle répondait, ou la réception répondait. On avait tous la même plateforme et tu voyais ce que les autres faisaient, donc on répondait dans les temps, mais dès que tu passais $19 \mathrm{~h}$ ou le week-end la charge de réponses était multipliée par 10, donc ça permet aussi d'analyser, donc c'est pas du tout pour les blâmer.

"Quand tu es à la réception ou au restaurant, tu dis vraiment Ah mais on me contrôle et en fait le management, je ne vais pas dire qu'il s'en fou du combien de temps tu as mis à la personne pour servir son café, mais c'est plus du coup comment fait on peut améliorer ça et où sont les problèmes. En tout cas moi c'est comme ça 
que je le vois. Ce n'est peut-être pas le cas de tout le monde, mais c'est vrai que la technologie, ça te permet de tellement tout contrôler.» (MDP, extrait d'entretien $n^{\circ}$ 9)

\subsubsection{Assurer la proximité avec l'équipe de réception par l'autonomisation et la responsabilisation}

Dans un système où le management se réalise par projet, où les amplitudes horaires couvertes ne permettent pas aux managers une supervision systématique et continue de l'activité, la solution trouvée par les MDP pour maintenir la proximité est la responsabilisation à travers l'attribution de missions spécifiques. Ces missions vont être supervisées en grande partie par le biais des outils digitaux. Les managers délèguent alors des projets qu'on leur impose et des responsabilités. Cela semble nécessaire pour se libérer un temps précieux pour d'autres tâches de fonctionnement (planification des travaux de l'hôtel, réalisation des plannings, préparation des entretiens annuels d'évaluation, etc.).
«À la réception, vous avez des jeunes, ce sont des gens qui ont envie d'apprendre, des gens qui ont soif, qui ont faim, alors il faut les nourrir. À ce moment-là, je me suis dit ok, il y a tellement de choses à faire donc je vais donner un projet à chacun [...]. J'ai chargé un réceptionniste de s'occuper d'un événement que l'on a à la fin du mois. C'est un truc pour les clients All et du coup on doit faire un événement. Elle s'occupe de tout, choix de la date organisation mise en pratique, je lui donne un budget et go. Et c'est très bien car elle du coup elle apporte des idées, elle se sent investie, ça change aussi de ce qui est habituel, ça me libère énormément de temps ! C'est juste que je suis. Alors on en est où ? On regarde ce que tu as fait... » (MDP, extrait d'entretien $n^{\circ} 10$ ).

41 C'est le cas, comme lors de l'organisation de soirées événements, pour la création d'un nouveau programme de fidélité. « Volontaires » et « convaincus» en tant que porteurs de projet, les réceptionnistes seront d'autant plus convaincants auprès des clients au moment de vendre ce nouveau service. Ainsi, la responsabilisation sur des projets de ce type permet plutôt que de donner « du sens » au travail des réceptionnistes, d'indiquer « la direction » que les équipes doivent suivre et intégrer dans leurs activités de travail dans les semaines, mois ou années à venir.

Devant la difficulté de pouvoir tout gérer, mobilisés par les systèmes d'informations et les tâches administratives de reporting, les managers de proximité s'éloignent de l'activité de management de la réception en consacrant moins de temps à l'encadrement et au soutien des réceptionnistes. Une partie des managers choisissent les dispositifs collaboratifs numériques pour animer et faire vivre le service de réception. Certains font usage des plateformes numériques par la création d'un groupe "Whatsapp " " pour gérer le planning des réunions d'équipe, les absences, les aléas dans les situations de travail et favoriser la remontée des informations et des problèmes plus rapidement le plus souvent par message vocal. Pour d'autres, c'est plutôt le réseau social numérique d'entreprise (RSNE) de l'entreprise qui permet la remontée informationnelle à travers les discussions qui s'y jouent. Ces supports par lesquels les managers communiquent sont aussi un moyen de maintenir la vitalité et la proximité avec le collectif à travers des événements, des soirées, des jeux de rôles, le partage se faisant à la fois dans et en dehors du strict cadre du lieu de travail. Paradoxalement, les MDP présentent presque l'obligation d'opter pour la connectivité permise par les dispositifs digitaux pour pallier les difficultés et relever les défis imposés par la digitalisation. 

par le cadre gestionnaire dans laquelle la digitalisation s'inscrit. Cette activité se réorganise notamment à travers la régulation des situations non gérées par l'outillage gestionnaire, et de nouveaux modes de fonctionnement du service de réception plus soutenus par le digital.

\subsection{Analyse de l'activité du manager de réception dans le cadre du projet de digitalisation de l'accueil Hospitality mobile}

Cette partie analyse sous l'angle ergonomique l'activité des managers de réception et leurs marges de manœuvre pour donner du sens (Rouleau, 2016) à une autre forme d'hospitalité : celle médiatisée par les technologies digitales (Bobillier Chaumon et al., 2015). Une des hypothèses soutenue est que la qualité du service et de la relation de service entretenue en situation dépend directement des marges de manœuvre des managers pour construire le pouvoir d'agir des réceptionnistes. Plus ces marges de manœuvre sont grandes et plus les managers auront la possibilité de rendre les situations d'accueil vectrices d'une bonne santé pour les réceptionnistes. Cette analyse implique en amont une analyse de l'activité des réceptionnistes.

Les résultats présentés sont issus prioritairement des observations des 3 réceptions des hôtels $\mathrm{A}, \mathrm{B}, \mathrm{C}$, des suivis d'activité des managers de réception et des entretiens complémentaires. Ils portent sur une analyse de l'activité des MDP dans l'accompagnement d'un projet de digitalisation de l'accueil Hospitality mobile. Ce projet a vocation de modifier le mode d'accueil des clients avec des réceptionnistes libres de leurs déplacements dans le hall de la réception, munis d'un téléphone multifonction («smartphone ») et en proximité des clients (Figure 3). 
Figure 2 : Check-in réalisé par un réceptionniste (droite) avec Hospitality mobile dans le hall. Figure 2: Check-in by a receptionist (right) with Hospitality mobile in the lobby

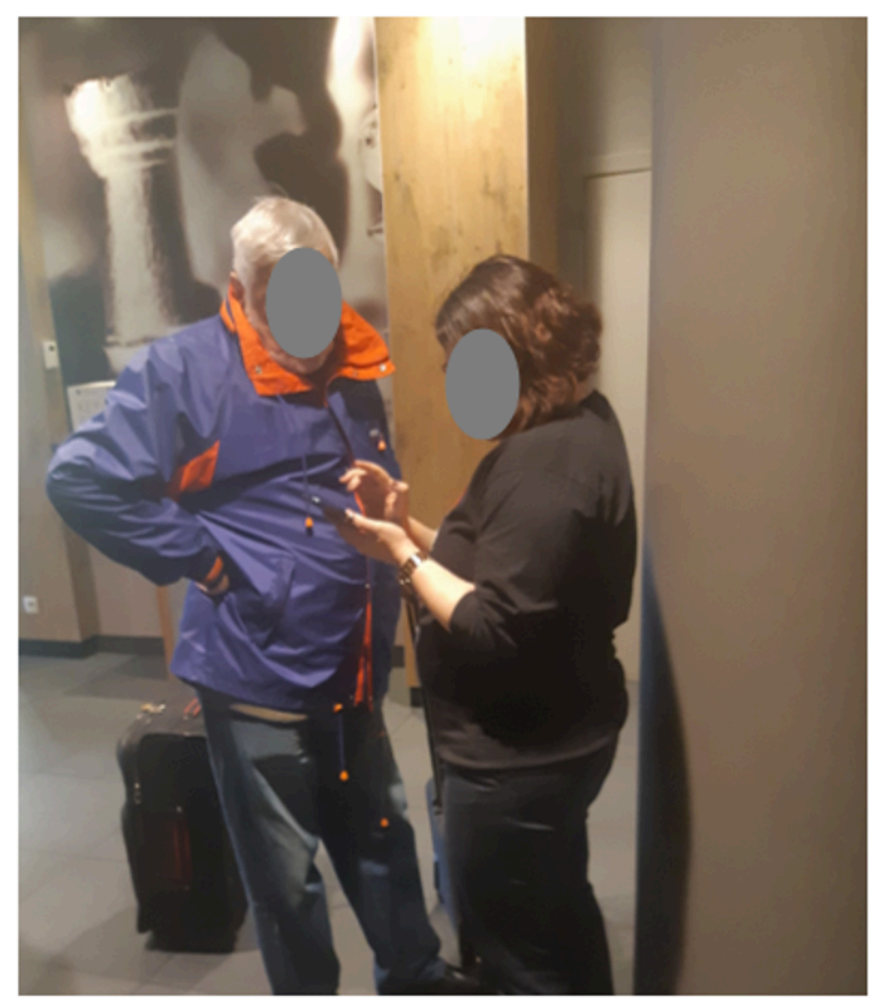

Ce mode d'accueil a pour objectif de se substituer au mode de réception classique (accueil au comptoir par les réceptionnistes). Le dispositif Hospitality mobile se veut aussi être un dispositif smartphone interopérable, utilisable comme moyen de communication et comme relevé d'informations entre réceptionnistes ainsi qu'entre les différents services de l'hôtel (housekeeping, maintenance, restauration). Ce dispositif et ses applications permettent un suivi en temps réel de l'activité dans l'hôtel au travers d'indicateurs tels que l'occupation des chambres, la propreté des chambres, etc. Lors de nos observations, les trois hôtels ne présentaient pas le même niveau d'avancement sur ce projet. Pour l'hôtel franchisé, le dispositif était déjà totalement intégré dans les pratiques d'accueil des clients. Pour les deux hôtels managés, il était encore en phase d'expérimentation et pas vraiment accepté ni adopté par les équipes de réception.

À notre arrivée, nous avions la connaissance d'un dispositif présentant une mauvaise évaluation suite à une première évaluation ergonomique. La présence d'un ergonome chercheur était pour les managers des hôtels A et B considérée comme une opportunité de réfléchir à l'évolutivité de l'organisation du travail. Le positionnement initial auprès d'un réceptionniste responsable syndical de l'hôtel a permis d'identifier les éléments bloquants de l'intégration de cette technologie.

\subsubsection{Comprendre les enjeux pour la réception du projet de digitalisation de l'accueil pour situer le rôle des managers de réception dans son accompagnement}

L'analyse de l'activité des réceptionnistes avant et après l'intégration d'Hospitality mobile est une étape essentielle dans la compréhension des agissements des managers de réception, de leur positionnement et des rôles qu'ils sont amenés à prendre. 

l'activité révèlent un regard partagé, perplexe des réceptionnistes sur Hospitality mobile quant à sa réelle plus-value, comparativement à une réception classique. L'expression consentie est celle d'un dispositif relativement peu polyvalent, limité à une vision élémentaire de l'accueil. Une réalité de terrain très différente selon chaque hôtel qui trouve ses origines selon la période du "shift $»^{14}$, le type de clientèle (business, famille, loisir), les spécificités organisationnelles de chaque réception au niveau de la répartition entre tâches administratives de «back-office » et tâches en "front office », et le nombre de tâches et de services (vente, distribution, surveillance) proposés qui peuvent venir se juxtaposer à la situation principale d'accueil. Dans certains cas, le dispositif est même contraignant pour la réalisation de certains types de tâches qui se présentent très fréquemment dans l'activité des réceptionnistes et qui participent pourtant, de la construction d'une continuité de la «châne de service» (Petit, 2005). Ces différentes composantes de l'activité ne sont parfois pas prises en charge par le dispositif smartphone. Plusieurs impossibilités ou difficultés fonctionnelles dans le cadre de nos observations sont apparues :

- Réaliser des walkins (client se présentant sans réservation préalable)

- Gérer les groupes-roominglist

- Verser des arrhes-Imprimer des factures, documents

- Modifier le stock dans le logiciel de point de vente (POS) (spécifique aux hôtels A et C)

- Aller sur Booking, Expedia

- Renseigner le take away

- Gérer les mails

Nous illustrons (Tableau 3) une situation d'accueil gérée difficilement avec Hospitality mobile: «Clients-touristes arrivant ensemble, mais dont chacun est muni d'une réservation individuelle»

Tableau 3 : Verbalisation post-activité, mise en évidence des régulations et des exigences de la situation d'accueil collective.

Table 3: Post-activity verbalisation, highlighting the regulations and requirements of the collective reception situation

\begin{tabular}{|c|c|c|}
\hline observation & $\begin{array}{l}\text { Bibliothèque de } \\
\text { situation }\end{array}$ & Retour sur l'activité post-situation \\
\hline $\begin{array}{l}\text { Situation } \\
\text { d'accueil } \\
\text { d'un groupe } \\
\text { de clients }\end{array}$ & $\begin{array}{l}\text { clients-touristes } \\
\text { arrivant ensemble } \\
\text { mais présentant } \\
\text { chacun une } \\
\text { réservation } \\
\text { individuelle }\end{array}$ & 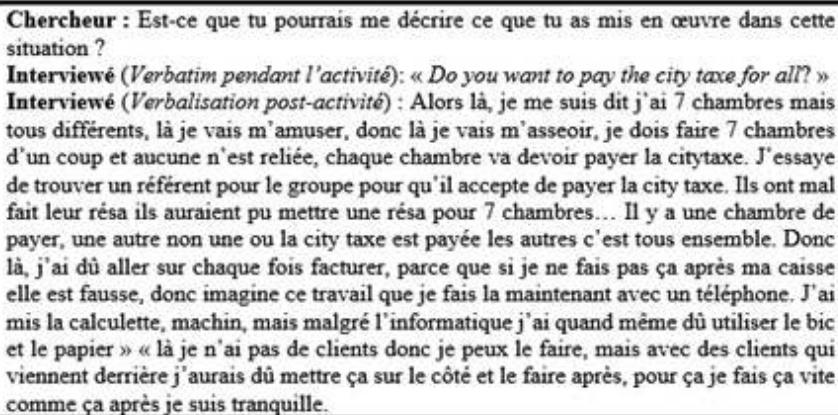 \\
\hline
\end{tabular}

51 On constate que Hospitality mobile ne permet pas la régulation des situations d'accueil. Il ne couvre pas l'ensemble des tâches de front office et back-office, et ne permet pas pour le moment le développement d'une marge de manœuvre situationnelle qui permettrait le choix du mode opératoire d'accueil des clients. Les nombreuses situations observées avec un flux élevé de clients témoignent notamment d'une difficulté à garder le 
contrôle sur son activité et la nécessité de devoir se replacer derrière le desk pour une gestion optimisée et ordonnée des files d'attente.

«Parfois quand il y a un rush, tu ne sais pas être devant le client... faire ça comme tu voudrais, quand t'es entouré t'es pas conscient de ça, alors que quand tu vois depuis ta réception, t'as une vue globale, tu vois ce qui se passe. Tu sais un peu mieux gérer les groupes que quand tu es entouré de tout le monde" (réceptionniste, extrait de verbalisation $n^{\circ} 11$ ).

Un autre exemple de difficulté observée chez un réceptionniste expérimenté de l'hôtel C, c'est l'analyse d'une situation d'accueil en l'apparence sans pression humaine (i.e. sans attente physique de client) qui révèle aussi que l'activité distancielle des clients sur les plateformes de réservation OTA («online travel agency») est génératrice d'une pression temporelle autant pour les réceptionnistes que les managers, amenés eux aussi par moment à réaliser des périodes courtes de check-in.

«Par exemple demain j'ai 0 chambre, mais je peux tricher et faire croire à l'ordinateur que j'ai une chambre en plus et au moment où je fais ça je dois me dépêcher parce que Booking.com, Expedia ils peuvent réserver à ce moment-là. Donc je fais ça, mais une fois que j'ai fait ça sur ce type de chambre là je dois faire l'inverse sur celle-là. Et donc ça c'est un truc que l'on fait souvent » (Verbalisation post activité d'un réceptionniste, extrait de verbalisation $n^{\circ} 12$ ).

Parfois même, l'application Hospitality mobile ne prend pas en compte la diversité des tâches à réaliser à la réception comme la possibilité de passer en overbooking qui offrirait la possibilité de disposer en situation de plus de marges de manœuvre quand il s'agit d'accueillir un ou plusieurs clients venus sans réservation.

«La fonction overbooking est une fonction primordiale que l'on doit avoir, mais on ne l'a pas sur le smartphone. C'est une fonction qui est obligatoire que l'on utilise tous les jours tous les shifts on est obligé de modifier les disponibilités » (réceptionniste, extrait de verbalisation $\mathrm{n}^{\circ} 13$ ).

L'objectivation sur une courte période de la plainte exprimée par les réceptionnistes, qui affirment que le dispositif ne permet pas réellement de personnaliser l'accueil, est justifiée par une plus grande attention visuelle portée sur le smartphone. Le partage d'attention entre le client et Hospitality mobile lors de deux séquences de check-in ( $1 \mathrm{~h}$ de film d'activité, Figure 4) montre que le réceptionniste de l'hôtel C utilise Hospitality mobile uniquement lors du deuxième check-in et il ne se déplace vers le client, dans aucune des deux séquences. L'analyse est aussi révélatrice du besoin de l'ordinateur en plus du smartphone pour encoder certaines informations et finaliser le check-in. Le constat est un non-déplacement des réceptionnistes vers le client, un partage de regard entre l'outil et le client allant légèrement en faveur de ce dernier. 
Figure 3 : Partage attentionnel ${ }^{15}$ (en \% de temps cumulé) lors de deux séquences de check-in. Figure 3: Attentional sharing (\% of cumulative time) during two check-in sequences

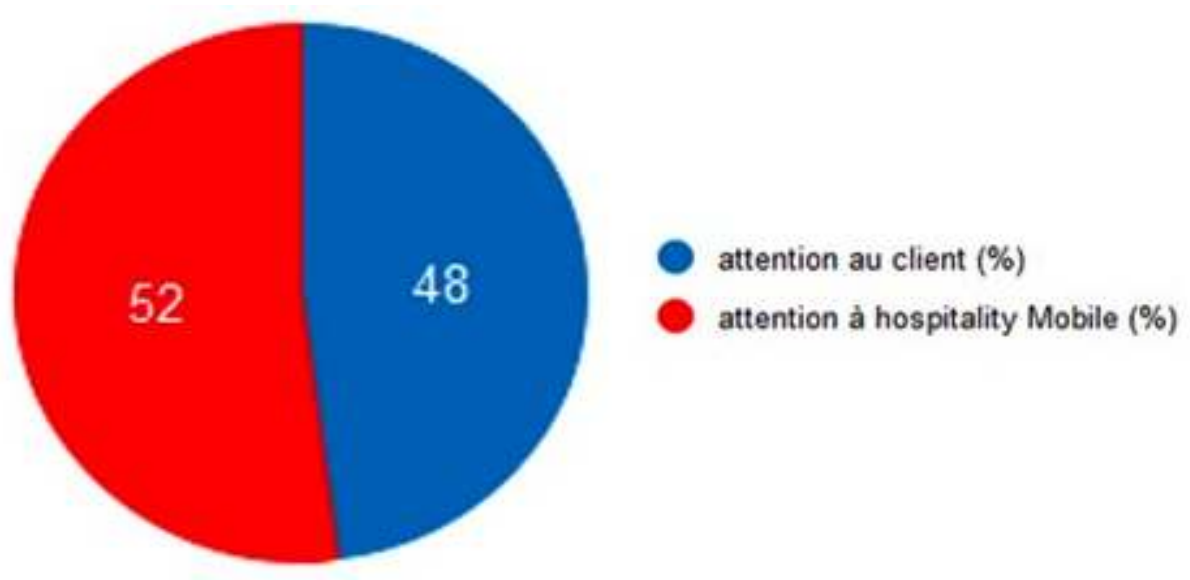
perçoivent le dispositif comme une menace pour la qualité de service ainsi qu'une remise en cause de leur identité de métier et de leurs savoir-faire. Cette question relative au sens du travail fait ressortir un dispositif qui porte dans son usage des conflits de buts importants, entre buts issus d'une logique du service au client et buts commerciaux. Pointée du doigt également, la nouvelle conception du métier de réceptionniste ou "Welcomer", tournée davantage vers une logique commerciale comme l'assume ouvertement les planneurs également concepteurs techniques d'Hospitality mobile.

"Au lieu de passer 5 minutes à faire de l'administratif on a 5 minutes pour expliquer au client la ville, l'hôtel, les services fournis par l'hôtel et donc du coup promouvoir des services payants, etc. On est beaucoup moins dans l'administratif et on est plus dans le commercial. On est dans le guest relation et ça c'est important ». (Directeur IT, extrait $n^{\circ} 14$ ).

Cet impact connu des concepteurs sur le travail de réception, dérange les réceptionnistes "expérimentés " qui ne trouvent plus de sens à leur activité initiale tournée vers la relation de service. Un autre effet qui ressort avec Hospitality mobile chez ces travailleurs expérimentés est la perte de la dimension collective de l'activité. L'environnement de coopération proxémique initial permettait une régulation transverse des situations d'accueil ou notamment l'écoute d'un pair, même sans interaction verbale représentait une donnée importante pour la production, mais aussi la reproduction d'un service de qualité, à travers l'espace d'apprentissage que cet espace constitue pour les nouveaux réceptionnistes. (Figure 5) 
Figure 4 : Environnement de coopération proxémique des réceptionnistes existant avant l'intégration d'Hospitality mobile.

Figure 4: Proxemic cooperation environment of receptionists existing before the integration of Hospitality mobile

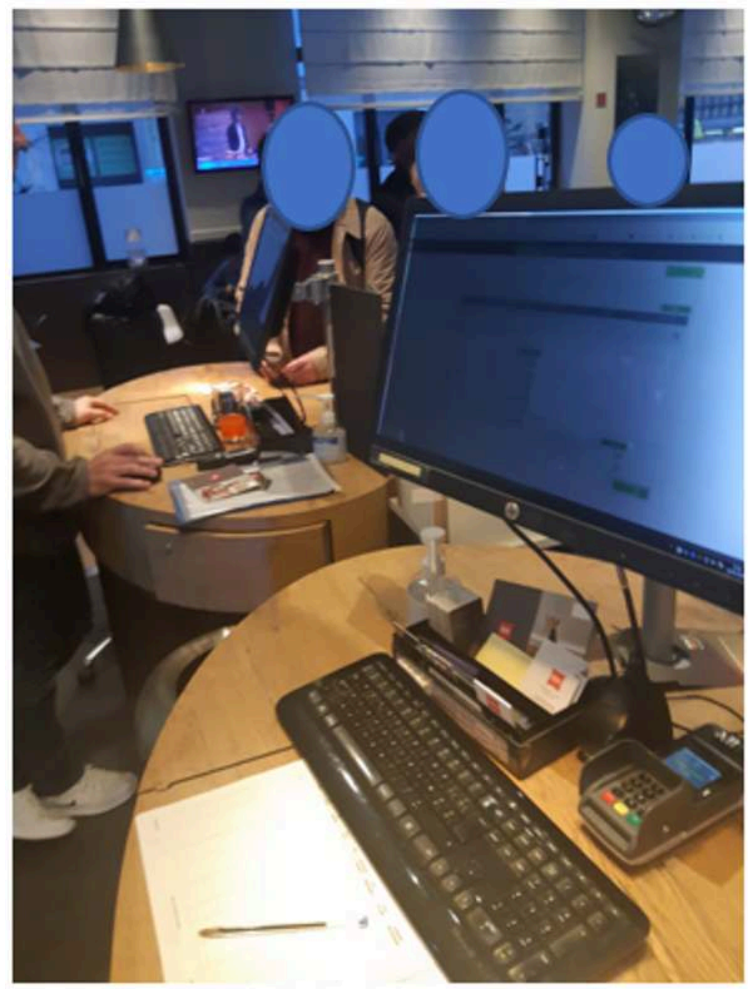

Pour le MDP de l'hôtel B, l'arrivée d'Hospitality mobile donne lieu à des réajustements de l'organisation du travail dans l'urgence, le plus souvent conscientisés, mais non anticipés par les concepteurs venant mettre à mal le mode d'organisation propre à chaque hôtel et de surcroît la capacité du manager à faire valoir ses choix organisationnels. Pour ce manager ce choix était un moyen d'éviter la répétitivité de la tâche et d'amener de la variabilité sur le temps du shift.

«Il faut vraiment réorganiser le boulot pour qu'effectivement le travail back-office soit fait en back-office et que le travail en front office soit fait en front office. (...) donc c'est une question d'organisation et une question de mentalité et de mindset vis-àvis de soi-même, mais également vis-à-vis du client. » (MDP, extrait d'entretien $\left.n^{\circ} 15\right)$.

\subsubsection{Une diversité de projets digitaux sur l'objet « accueil » et des managers pour redonner une vision systémique au changement}

\section{L'articulation des projets et la mise en cohérence du projet de digitalisation}

Dans les grandes chaines hôtelières comme GRZ, de nombreux projets de transformation, le plus souvent digitaux, se superposent obligeant les managers à jongler, arbitrer, prioriser. Le cas de l'accueil est typique avec 5 projets qui coexistent : Hospitality mobile, refonte architecturale du lobby, installation d'une borne cliente d'information digitale, projet "cashless " visant la suppression de toute liquidité, sans oublier le "fastcheckout» pour fluidifier les départs des clients. Autant de projets que les MDP doivent intégrer et aborder systémiquement « comme un tout » pour tenter de garder une cohérence vis-à-vis des collaborateurs et la confiance de ces derniers dans 
les choix d'accueil. Les managers font preuve d'habilité en repensant Hospitality mobile à travers des projets parallèles, comme la reconception architecturale du hall potentiellement plus riche en marge de manœuvre pour agir. C'est ainsi que l'organisation d'ateliers de travail, planifiés, organisés sur des plans architecturaux, animés par le manager lui-même, est le moyen utilisé pour s'approprier et laisser s'exprimer le travail et l'idéation collective. Ils nous expliquent que réfléchir à l'emplacement du "terminal de paiement ", de «l'imprimante », des «badges » c'est très vite en arriver à l'évocation des situations concrètes de travail et retrouver cette proximité avec les équipes.

\section{L'articulation du social et du digital pour maintenir l'efficience du service de réception}

L'activité des MDP dans ces hôtels s'inscrit dans une histoire marquée au moment de notre présence par des relations conflictuelles entre les syndicats et la direction. L'ancrage des managers chez GRZ se réalise dès le recrutement pendant lequel les MDP acceptent ce rôle d'agent du changement qui leur est assigné. Quand il s'agit de l'assumer, les managers l'exercent dans chaque organisation avec plus ou moins d'engagement et de conviction, confrontés chacun à des contextes sociaux spécifiques dans lesquels se jouent des jeux de pouvoir associés au projet Hospitality Mobile et une nouvelle conception du métier de réceptionniste. Prendre en compte les différentes logiques est d'autant plus important que ces acteurs sont amenés à se rencontrer au moment des groupes de travail.

«On a des réunions tous les mois avec les syndicats, les responsables de chaque hôtel pilote, les responsables d'application pour améliorer tous ensemble, c'est un plan de travail ». (MDP, extrait d'entretien $\left.n^{\circ} 16\right)$.

\section{Un contrôle faillible d'Hospitality mobile qui préserve des marges de manœuvre}

En dehors de ce temps formel de réunion où priment les enjeux de chacun, le travail continue à se réaliser si bien que cela se traduit au niveau des MDP, par des prises de décisions agiles par leur connaissance des limites du dispositif de surveillance d'Hospitality mobile. Les managers ont notamment acquis un savoir précieux des critères utilisés pour évaluer Hospitality Mobile restreignant du même coup, le pouvoir disciplinaire du dispositif et le côté prescriptif de son usage dans la situation d'accueil. Ainsi, bien que contrainte par des règles de contrôle, la méthode de mesure de l'usage d'Hospitality mobile permet aux MDP de bénéficier de marges de manœuvre dans le choix de la méthode de travail. Cette connaissance permet la préservation des espaces d'autonomie aux réceptionnistes. La possibilité est ainsi laissée à chaque réceptionniste d'œuvrer soit avec le dispositif d'accueil classique (Figure 6) soit avec Hospitality mobile puisque «les $K \mathrm{KI}^{16}$ ne prennent uniquement en compte que les check-in et checkout » (MDP hôtel B). 
Figure 5 : Illustration d'un poste avec ordinateur fixe et pivotable muni de Hospitality Mobile. Figure 5: Illustration of Swivel computer with Hospitality Mobile

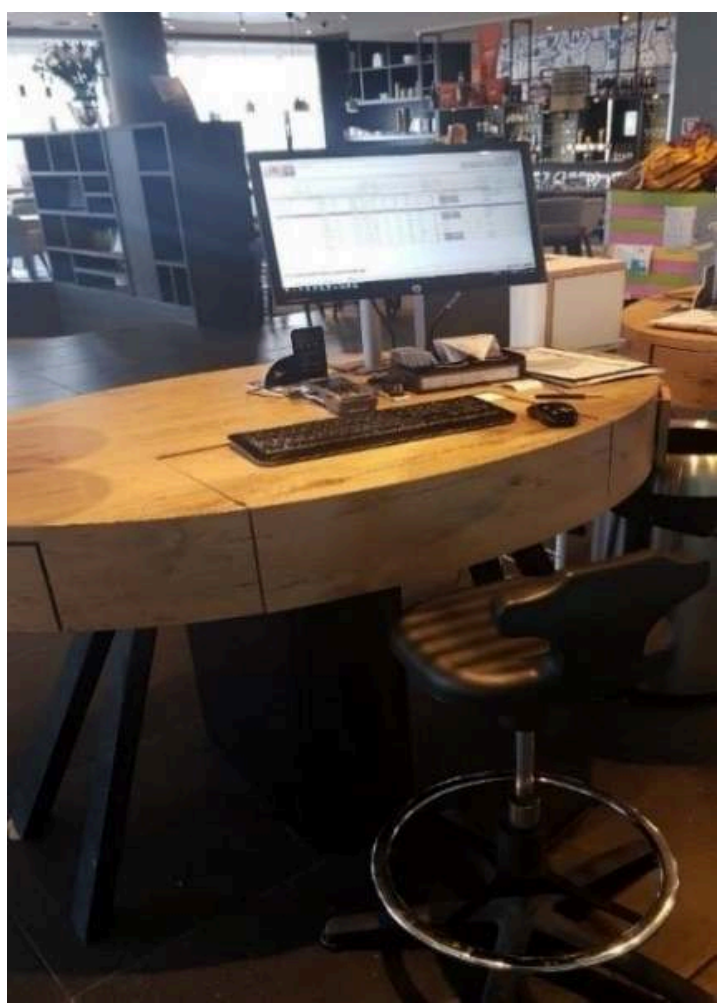

61 Pouvoir jongler entre les deux dispositifs représente la solution trouvée pour maintenir une qualité d'accueil suffisante, un bon climat social. Concrètement, cela se traduit par des managers conciliant avec les réceptionnistes les plus réfractaires, notamment certains représentants syndicaux. Ils essayent en contrepartie d'inciter "gentiment » les réceptionnistes nouvellement arrivés et les moins "transgressifs" (Babeau \& Chanlat, 2008) à expérimenter Hospitality mobile pour en faire les relais actifs de la transformation digitale et atteindre les $90 \%$ de cas traités avec le dispositif. Les managers adoptent des comportements conformes par rapport aux attentes managériales contenues dans les indicateurs les plus étroitement surveillés. Cependant, cette régulation et l'élaboration de cette nouvelle règle de travail amène aussi à des comportements d'usage non sensés comme la réalisation d'un check-in de groupe qui se transforme en encodage individuel sur Hospitality mobile après la montée en chambre des clients, pour se rapprocher des objectifs de check-in mobile fixés. Ces compromis négociés entre le manager et l'équipe de réception sont nécessaires, mais renforcent chez certains le sentiment de faire un travail inutile, non sensé, délétère pour la santé, mais aussi la performance.

En laissant ces possibilités d'action aux réceptionnistes, les managers de proximité parviennent aussi à faire évoluer les règles implicites pour créer des nouvelles règles de travail. Ce mode d'action à l'interface des différentes logiques permet la rédaction de plans d'action plus réalistes et de créer des nouvelles règles sur les étapes d'implantation de Hospitality mobile. Les managers sont toutefois lucides que, de toute façon, tôt ou tard, ils devront se résoudre à se réinventer avec le dispositif.

« On sait que ça va devoir changer [...] S'ils veulent le forcer, ils le rajoutent dans les objectifs du directeur et le directeur va le répercuter sur le front. Le front va le répercuter sur l'exécution. Donc ça va venir, mais tranquille tant que ce n'est pas 
$100 \%$ ok qu'ils n'ont pas pensé à tout. En Belgique il y a les syndicats et s'ils estiment qu'il y a des choses qui n'ont pas été bien réfléchies ils vont stopper [...] » (MDP, extrait $\left.n^{\circ} 17\right)$.

\subsubsection{Des marges de manœuvre réduites sur la conception}

\section{La formation, support de l'enrôlement des collaborateurs dans le changement}

Compte tenu des difficultés à rendre ergonomique le dispositif Hospitality mobile en raison de la forte dissociation entre les concepteurs du dispositif et les utilisateurs finaux, les managers entrent dans un rôle d'enrôlement. Cette posture vise à munir les réceptionnistes pour qu'ils puissent faire face à la nouvelle fonction de "welcomer » et aux situations d'accueil qu'ils rencontreront. Les MDP se tournent vers les organisations professionnelles, les formations internes nombreuses avec pour cible la situation relationnelle réceptionniste-client comme principal élément d'empowerment. La perspective de ces formations est de simuler un accueil client avec le smartphone et ainsi responsabiliser le réceptionniste dans ses choix d'interaction avec le client (positionnement, posture, langage). En aucun cas, ces simulations ne permettent de rendre compte de l'environnement dynamique existant à la réception, conçu sur un mode collaboratif entre réceptionnistes et qui plus est, est intimement dépendant des autres services.

«En Belgique il y a beaucoup de possibilités, formation interne, Horeca... Moi mes réceptionnistes 2 par mois ils sont inscrits sur ces formations. C'est vraiment le savoir-être qui est important. Mais même l'entreprise met plein de formations internes qui jouent sur les émotions sur l'être humain c'est vraiment la chose la plus importante [...] on a fait une formation interne tous les front office managers des hôtels pilote, on a été à Amsterdam qui ont depuis des années Hospitality mobile.» (MDP, extrait $\left.n^{\circ} 18\right)$.

\section{Des managers de réception qui donnent « le sens de l'hospitalité »} lesquelles ils peuvent apprendre les bonnes pratiques: comment se comporter avec chaque profil client, quel « body langage » adopter, comment garder l'esprit ouvert («le mindset ») ou encore, comment amener un client de manière "naturellement forcée " à ne pas demander une facturation papier. Cet objectif nécessite l'expression de compétences d'animation aux MDP. Des compétences managériales qui ont vocation à développer les ressources individuelles et à faire de réceptionniste des "Heartist " maitrisant l'art d'accueillir et de servir les autres avec cœur, curiosité et inventivité.

" Au début on a fait comme des jeux. Par exemple, le collaborateur se mettait dans la peau d'un client. On a fait vraiment vivre la situation avant que le portable arrive. (...) C'est vraiment les personnes qui ont vraiment du mal que je mettrais derrière à faire des contrôles et toutes les choses qu'on doit faire pour préparer l'arrivée des clients. » (MDP extrait $\left.\mathrm{n}^{\circ} 19\right)$.

Plus que de donner un sens à la situation d'hospitalité, les MDP enrôlent les réceptionnistes en mobilisant leur sens de l'hospitalité. Dans ces formations, le développement des compétences individuelles axées sur des dimensions de créativité, d'expressivité, prime sur les déterminants organisationnels de la situation d'accueil. Il nous parait que grâce à cet axe de formation orienté sur la formation aux émotions, les réceptionnistes se trouvent moins démunis, et mieux préparés individuellement. Ils présenteraient moins de raisons d'opposer une résistance à l'usage d'Hospitality mobile. 
Le smartphone s'ancrerait de cette façon dans les pratiques devenant un outil médiateur de l'activité comme les autres. Cette orientation n'est cependant pas sans effet puisqu'elle génère de nouvelles orientations dans les parcours de travail avec des réorientations des postes de la réception vers des postes plus administratifs moins dans la relation de service au client comme celui de la gestion des réservations.

\section{Discussion}

La diversité des séquences d'activité traitées nous a permis d'identifier des composantes des conditions du travail réel des managers de proximité permettant de décrire et de caractériser cette activité. Nous revenons dans cette discussion sur l'expression de certains « rôles » observés dans le cadre des suivis d'activité.

\subsection{La fin du contrôle managérial, entre expression traditionnelle des rôles et besoin de modernité ?}

On aurait pu s'attendre à trouver des managers libérés des tâches de reporting et du contrôle du travail des employés (Abbasi et al., 2016). Le contrôle exercé par les outils de gestion rejaillit au contraire sur les MDP par une exacerbation du contrôle sur les équipes de réception par le biais des outils digitaux mis à disposition par le groupe hôtelier (RSNE, smartphone, PMS). Nous retrouvons des MDP soumis à une profonde instabilité, ballotés entre les rôles traditionnels focalisés sur le respect des règles, la recherche d'efficacité et d'efficience (Attarça, \& Chomienne, 2014 ; Desmarais, \& Abord de Chatillon, 2008, 2010).

Globalement, les MDP font part de la mise en place d'une multiplicité de dispositifs de contrôle numérique relatifs au temps, aux lieux ou encore au contenu de leur travail. Ils évoquent de la sorte des situations de stress et de perte de confiance liées aux possibles usages déviants des outils numériques en tant que dispositif de surveillance ou de dispositifs disciplinaires. En distribuant les responsabilités sur des projets, les managers parviennent à prendre de la distance tout en maintenant la proximité d'un suivi à distance, supervisé par le biais des outils digitaux (Schmitt, Fabbri, \& Gallais, 2011). L'engagement des collaborateurs dans la stratégie digitale de l'organisation par la responsabilisation et l'autonomisation semble être malgré tout, la principale stratégie développée par les MDP pour faire face à un système inadapté au travail : aussi bien le leur que celui des réceptionnistes.

\subsection{La mise en visibilité du travail auprès des pilotes : donner à voir l'activité déployée à la réception}

Très précis sur les résultats attendus, le système de pilotage se fonde principalement sur des outils de gestion (PGI, GRC) et algorithmes intelligents, révélant un flou sur les moyens d'atteindre le niveau d'efficience attendu. Cela se traduit en termes d'activité managériale par un effort constant d'ajustement et un important travail de traduction (Desmarais, \& Abord de Chatillon 2010) auprès de l'équipe de réception. Un rôle qui vise à «articuler des logiques contradictoires, à leur donner du sens, à les intégrer au sein d'un cap flexible et évolutif en fonction des pressions subies et des ressources développées pour résister à ces pressions» (Desmarais, \& Abord de 
Chatillon, 2010, p. 77). Le travail de traduction ne se limite pas ici à la sphère d'exécution du travail, il s'oriente également vers les revenue managers. Les MDP s'autoprescrivent des temps de reporting pour combler l'inefficacité de ces systèmes à saisir l'activité déployée des collaborateurs, reporting nécessaire pour libérer des marges de manœuvre collectives pour les acteurs de la réception et pour lui-même.

\subsection{Le manager de réception au cœur du travail d'organisation}

La situation évoquée concernant le départ prématuré du client fidèle, montre l'intervention des MDP dans le cadre d'une situation insolvable par des algorithmes. Observée dans l'action, elle reflète une certaine inefficacité des dispositifs numériques déployés à saisir la complexité du travail dans une situation imprévue. Un travail complexe qu'il faut réaliser en tenant compte de plusieurs objectifs parfois contradictoires. Les MDP éprouvent une certaine utilité et se réapproprient des données pour soutenir leur activité dans leurs prises de décision au moment de résoudre des dilemmes non prescrits par l'organisation. En l'occurrence ici, l'assurance de voir un client régulier satisfait. Malgré cet usage du digital, nous situons cette situation comme allant à l'encontre des bénéfices de l'«automatisation de la gouvernance" (Jentzsch, 2016) pour dépasser les "négociations humaines désordonnées et inefficaces " (Lee et al., 2015 ; Newell \& Marabelli, 2015). On retrouve ici un travail d'organisation des MDP orienté vers celui de la régulation (Dujarier, 2006). Autrement dit un travail "de conception de la prescription, mais aussi de ses renormalisations successives, dans un travail de régulation " (Reynaud, 1988), « une production normative, résultant de la mobilisation d'acteurs multiples » (de Terssac, 2011). Ici encore, c'est la dimension collective qui est source d'efficacité et de réalisation d'un travail qui pourrait être vu comme de la sur-qualité, mais qui fait en réalité partie du quotidien des équipes de réception. De notre point de vue, cette situation illustre que l'efficacité de ces systèmes est liée à la capacité qu'ils ont de soutenir et de faciliter la prise de décision en tant que manager. Ce soutien semble prendre sens lorsqu'ils sont vus par les managers comme complémentaires à l'activité initiale qui est celle de la personnalisation du service et qu'ils permettent une gestion collective de la personnalisation.

\subsection{Du cadre d'action au cadre pour l'action dans l'usage d'Hospitality mobile}

\subsubsection{L'articulation des enjeux du projet digital d'accueil : une activité de régulation}

Si l'organisation définit le cadre d'action, les managers font en sorte de créer un cadre pour l'action (Gotteland-Agostini, Pueyo, \& Béguin, 2015) qui permet l'exercice de la régulation. Dans ce processus de management du changement technologique, on constate que la régulation de contrôle (celle qui imposée un pourcentage d'utilisation d'Hospitality mobile) qui regroupe "l'ensemble des règles qui indiquent aux salariés comment ils doivent travailler et coopérer" (Havard \& Krohmer, 2008), est réinterprétée selon les contextes sociaux et les jeux d'acteurs (syndicats, réceptionniste novice, réceptionniste). Pour parvenir à un consensus d'usage, les managers doivent arbitrer différents enjeux, différentes subjectivités, différentes visions sur le travail de 
réceptionniste. Il s'agit de mettre en confrontation et d'articuler les points de vue, des logiques professionnelles divergentes, voire contradictoires qui n'ont aucune raison de coexister (Dugué, Petit, \& Daniellou, 2010). Sans s'en apercevoir, les MDP sont en train d'encadrer le travail à travers un processus de régulations conjointes, c'est-à-dire un ensemble de "négociations implicites faites d'arrangements tacites [qui] forment le vrai du travail d'organisation » (de Terssac, 2011 ; de Terssac, Lalande, \& Tremblay, 2003) dans le cœur de l'activité collective.

\subsubsection{Un effort d'enrôlement pour rendre acceptable le dispositif Hospitality mobile}

72 Les MDP font en sorte de permettre aux collaborateurs d'interpréter la teneur des changements opérés et de produire du sens sur ces changements, notamment en adoptant un rôle exemplaire. Nous avons repéré des stratégies de rapprochement chez les managers, qui n'hésitent pas par intermittence à utiliser eux-mêmes le dispositif Hospitality mobile pour tenter d'être en proximité par le rôle de soutien de leur équipe au moment des roulements, des périodes de rush (Piney, 2015). Ils jouent ainsi la carte de la proximité technique légitimée par une pratique professionnelle antérieure de réceptionniste pour enrôler les collaborateurs dans le changement digital. Par ce processus, ils deviennent de véritables acteurs du changement. Malgré des marges de manœuvre réduites, les managers mettent de l'énergie pour donner du sens (Rouleau, 2016) à cette autre forme d'hospitalité médiatisée par les technologies digitales (Bobillier Chaumon et al., 2015). L'extrait $n^{\circ} 18$ évoquant le développement des compétences individualisantes montre que les MDP font en sorte de donner les capacités à leurs équipes de «bien vivre » ou de « mieux vivre » le changement digital, et comment ils arrivent à agir "sur le projet de changement lui-même et sur la signification qui lui est attribuée » (Rouleau \& Balogun, 2007). La priorité est mise sur la formation aux soft-skills pour favoriser l'empowerment (Benedetto-Meyer, 2019a, 2019b) des réceptionnistes dans la situation d'hospitalité future. La dynamique de la construction du sens par les MDP et le travail d'enrôlement sont mis en place à travers une orientation vers la recherche de l'allocation des ressources aux réceptionnistes qui correspond au travail de «leader habilitant» déjà démontré dans le secteur de l'aéronautique (Caillé \& Jeoffrion, 2019).

\section{La digitalisation de l'hôtellerie : quelles possibilités de développement de l'activité managériale?}

Les usages des technologies ne sont pas neutres. En regard des recherches qui annonçaient une "révolution" ou "disruption" des contextes organisationnels (Barton \& Court, 2012) et du «management» (Gobble, 2013), notre analyse met en évidence que la digitalisation s'inscrit dans le prolongement de l'installation d'un cadre gestionnaire de travail qui était déjà en pleine implantation dans l'hôtellerie et que la digitalisation vient renforcer. La vision techno-pessimiste des MDP en tant que mise à distance de l'acte même d'hospitalité "happé par les tâches concurrentes" (Detchessahar, 2013) orientée vers «l'alimentation des machines de gestion» (Girin, 1990) semble trouver une forme de validation dans notre étude de cas à travers ce que nous avons défini comme la "gestionnarisation" de la relation de service. Grâce à la triangulation des méthodes de collecte de données et leur analyse approfondie, ces 
résultats paraissent valides et congruents. Toutefois, ils rencontrent les limites habituelles d'une étude de cas et ne peuvent prétendre à une généralisation. Les résultats nécessitent un étayage sur base d'autres études de cas dans d'autres structures hôtelières ou la digitalisation prend place dans des dimensions organisationnelles, culturelles et humaines différentes.

Cette étude permet de rapprocher l'activité du manager de réception de celle d'une activité soumise à un management algorithmique par l'IA «forcé librement » par les pilotes. Les algorithmes sont très présents dans la chaîne de valeur et poussés par les pilotes eux-mêmes, pour être mis en application directement dans les situations d'accueil. L'analyse permet de démontrer un certain pouvoir structurant des technologies sur l'activité. Cependant, l'activité du manager ne fait pas que réagir au prescrit, elle n'est pas simple exécution. Si la présence gestionnaire et algorithmique se fait bel et bien ressentir, la recherche est plus largement révélatrice des processus de régulation organisationnelle et humaine mis en œuvre par les managers. La plus-value de l'analyse ergonomique de l'activité a été de montrer des contournements face à ce management prescriptif souvent incomplet. Détournée, bricolée, l'activité managériale trouve son sens dans le besoin de contourner un certain nombre de limites, de dysfonctionnements ou autres désagréments occasionnés par les technologies digitales pour atteindre la performance attendue par l'organisation et celle qu'ils se sont fixés pour eux-mêmes. Un autre intérêt de l'étude a été de rendre compte que malgré la portée grandissante du digital, la relation de service client semble plus que jamais personnalisée par l'activité humaine de co-production avec les métiers de contact (Cerf, 2005 ; Valléry, \& Leduc, 2005), même si nous faisons également le constat que la digitalisation peut donner lieu à de nouvelles médiations au sein du collectif de réception, changer la nature de la tâche et impacter plus largement le système d'activité (Leontiev, 1984) dans lequel agissent les managers.

Concernant sa construction, l'étude a été l'occasion à travers la construction sociale (Coutarel, \& Daniellou, 2007) de proposer des pistes d'amélioration pour trouver des compromis et des situations moins délétères du point de vue des conditions de travail du manager de proximité et ainsi contribuer à une réflexion centrée sur l'activité de travail. Il s'agissait de se centrer spécifiquement dans les retours réalisés sur les régulations mises en œuvre par les MDP.

Pour ce qui est de la place des managers dans le changement digital avec l'intégration du dispositif Hospitality mobile, nous avons développé le rôle actif des managers de réception malgré des marges de manœuvre réduites sur sa conception (facilité d'usage, utilisabilité). Nous avons contribué à décrire les reconfigurations de l'activité en montrant des managers de réception habiles pour négocier avec leurs équipes les nouvelles conditions de réalisation du travail à travers la régulation conjointe (Reynaud, 2003) dans le type d'usage d'Hospitality mobile, mais aussi pour leur donner les moyens de «faire face » aux transformations du métier de réceptionniste apportées par les stratégies de digitalisation. De ces marges de manœuvre, liées aux failles de surveillance du système, découle une posture managériale parfois ambivalente. L'analyse de l'activité a permis d'identifier et de comprendre où se situent ces marges de manœuvre pour donner du sens (Rouleau, 2016) à une autre forme d'hospitalité médiatisée par les technologies digitales (Bobillier Chaumon et al., 2015). Les managers ont ainsi développé des rôles émergents notamment pour actionner différents leviers 
d'acceptation du dispositif Hospitality mobile. Nous retenons en particulier, l'adaptation des ressources individuelles par des mises en situations.

De notre point de vue, plutôt qu'un réel développement du pouvoir d'agir des réceptionnistes avec le dispositif, l'activité managériale dans ce changement s'apparente plutôt à un rôle d'«empowerment». Les managers cherchent le développement de marges de manœuvre externes (Coutarel et al., 2015) à la situation d'accueil à travers un enrichissement des compétences attitudinales. Ce travail sur l'émotion ou travail émotionnel comme il a été proposé par Giacomel (2019), nous semble trop limité dans des projets aussi disruptifs et impactant pour le sens du travail. Une perspective développementale qui peut trouver une efficacité sur du court terme, mais éloignée de la vision d'une ergonomie de conception à visée capacitante (Falzon, 2013). Plusieurs ingrédients pourraient selon nous jouer en faveur d'une digitalisation source mutuelle de développement du pouvoir d'agir des managers et des réceptionnistes. Un premier serait de "désempêcher les managers", en libérant leurs capacités attentionnelles, par une simplification des procédures, une meilleure définition de leur périmètre, une limitation d'information descendante et en améliorant les choix des indicateurs à reporter. Cela leur éviterait l'activité d'autoprescription extrêmement coûteuse. Si « l'empêchement » n'est pas caractéristique de l'ensemble des managers des hôtels A, B, C, cette recommandation peut être retenue comme piste. Dans la visée d'une ergonomie de conception, une autre piste consiste au rapprochement du monde des concepteurs du dispositif Hospitality mobile (chef de projet Hospitality mobile pour le groupe hôtelier au niveau monde), des usagers effecteurs du travail (réceptionniste, manager mais aussi les pilotes). La tentative actuelle que nous menons et qui semble être une voie porteuse (Rocha, Mollo, \& Daniellou, 2017) consiste à placer la personnalisation du service comme un objet frontière (Galey, Judo, Jolly, Goutille, Morelot, Albert, et al., 2019, p. 19) discutée sous le prisme de l'activité et plus particulièrement, sur celui de la qualité de la relation de service et ses redéfinitions dans ce nouveau contexte.

\section{BIBLIOGRAPHIE}

Abbasi, A., Sarker, S., \& Chiang, R. H. (2016). Big data research in information systems: Toward an inclusive research agenda. Journal of the Association for Information Systems, 17(2), 3.

Attarça, M., \& Chomienne, H. (2014). Les politiques publiques sous l'influence des entreprises. Revue française de gestion, 8, 101-130.

Autissier, D., Johnson, K., \& Moutot, J. (2015). De la conduite du changement instrumentalisée au changement agile. Question(s) de management, 10(2), 37-44 https://doi.org/10.3917/qdm.152.0037

Autissier, D., \& Vandangeon-Derumez, I. (2007). Les managers de première ligne et le changement. Revue française de gestion, 174(5), 115-130. https://doi.org/10.3166/rfg.174.115-130

Autissier, D., Vandangeon-Derumez, I., \& Vas, A. (2010). Conduite du changement : concepts-clés : 50 ans de pratiques issues des travaux de 25 grands auteurs. Paris : Dunod 
Babeau, O., \& Chanlat, J.-F. (2008). La transgression, une dimension oubliée de l'organisation. Revue française de gestion, 3, 201-219.

Bardin, L. (2013). L'analyse de contenu. Paris : Presses Universitaires de France. https://doi.org/ 10.3917/puf.bard.2013.01

Barton, D., \& Court, D. (2012). Making advanced analytics work for you. Harvard business review, 90(10), 78-83.

Benedetto-Meyer, M. (2019a). Les managers de proximité sont-ils les acteurs d'une forme d'empowerment? Terminal. Technologie de l'information, culture \& société, 125-126.

Benedetto-Meyer, M. (2019b). Le travail d'une manager face à la «transformation numérique ». Savoir/Agir, 1, 87-95.

Bobillier Chaumon, M.-E. (2021). Emerging Technologies and Issues for Activity and Occupational Health. Digital Transformations in the Challenge of Activity and Work: Understanding and Supporting Technological Changes, 3, 1-19.

Bobillier Chaumon, M.E. Sarnin P., Cuvillier, B., \& Vacherand-Revel, J. (2015). L'activité médiatisée des cadres par les technologies de nouvelles pratiques pour de nouvelles compétences? In C. Felion (Ed.), Les cadres face aux TIC : enjeux et risques psychosociaux au travail. Paris : L'Harmattan.

Bolduc, F., \& Baril-Gingras, G. (2010). Les conditions d'exercice du travail des cadres de premier niveau : Une étude de cas. Perspectives interdisciplinaires sur le travail et la santé, 12(3).

Brown, P., Ashton, D., \& Lauder, H. (2010). Skills are not enough: The globalisation of knowledge and the future UK economy.

Caillé, A., \& Jeoffrion, C. (2019). Effet des pratiques managériales habilitantes sur le bien-être psychologique au travail : Le rôle déterminant de la confiance et du sens au travail. In Psychologie sociale appliquée aux grandes thématiques contemporaines : Sécurité routière, religion, environnement, discrimination, travail (p. 171-183). L'Harmattan.

Camus, S., Hikkerova, L., \& Sahut, J.-M. (2010). Tourisme durable : Une approche systémique. Management Avenir, 4, 253-269.

Cerf, M. (2005). Situations de service: Travailler dans l'interaction. PUF.

Clot, Y., \& Simonet, P. (2015). Pouvoirs d'agir et marges de manœuvre. Le Travail Humain, 78(1), 31-52.

Coutarel, F., Caroly, S., Vézina, N., \& Daniellou, F. (2015). Marge de manœuvre situationnelle et pouvoir d'agir : des concepts à l'intervention ergonomique. Le travail humain, 78(1), 9-29. https://doi.org/10.3917/th.781.0009

Coutarel, F., \& Daniellou, F. (2007). Construction sociale du changement en ergonomie et réseaux sociaux : Vers une conduite de projet orientée "conception d'organisations capacitantes". Colloque Management et Réseaux Sociaux, Capital social et/ou réseau social en actes : quels outils de gestion ?, 85-86.

Cuny-Guerrier, A., Caroly, S., Coutarel, F., \& Aublet-Cuvelier, A. (2018). Les composantes de la marge de manœuvre situationnelle d'encadrants de proximité en situation de sous-traitance : Un enjeu pour la prévention des troubles musculo-squelettiques (TMS). Le Travail Humain, 81(2), 85-114.

Daniellou, F., Boissières, I., \& Simard, M. (2010). Les facteurs humains et organisationnels de la sécurité industrielle : Un état de l'art. FonCSI. 
Dehame-Leleyter, M., Lancry, A., \& Vallery G. (2008). Travail d'articulation et organisation de travail : Etude dans un collectif de l'enseignement public. Psychologie du Travail et des Organisations. $14(4), 345-368$

Denizci Guillet, B., \& Chu, A. M. C. (2021). Managing hotel revenue amid the COVID-19 crisis. International Journal of Contemporary Hospitality Management.

Desmarais, C., \& Abord de Chatillon, E. (2008). Existe-t-il encore des différences entre le travail des managers du public et ceux du privé ? Revue française d'administration publique, 4(128), 767-783.

Desmarais, C., \& Abord de Chatillon, E. (2010). Le rôle de traduction du manager : entre allégeance et résistance. Revue française de gestion, 205(6), 71-88.

Detchessahar, M. (2011). Santé au travail. Revue française de gestion, 5, 89-105.

Detchessahar, M. (2013). Management empêché, santé dégradée : Le blues des managers. Santé et travail, 82, 26-28.

Dugué, B., Petit, J., \& Daniellou, F. (2010). L'intervention ergonomique comme acte pédagogique. Perspectives interdisciplinaires sur le travail et la santé, 12(3).

Duhamel, S., Olry P., \& Cerf M (2021). Apprendre à déceler le potentiel de développement des situations de travail : l'exemple de conseillers agricoles face aux enjeux de l'agro-écologie. Activités, 18(1). https://journals.openedition.org/activites/5983

Dujarier, M. (2006). La division sociale du travail d'organisation dans les services. Nouvelle revue de psychosociologie, 1(1), 129-136. https://doi.org/10.3917/nrp.001.0129

Dujarier, M.-A. (2015). Le management désincarné. La Découverte.

Dujarier, M.-A. (2017). Le management désincarné: Enquête sur les nouveaux cadres du travail. La Découverte.

Eckert, H., \& Monchatre, S. (2009). Les carrières ouvrières à l'épreuve de la polyvalence, analyse de deux cas français. Revue multidisciplinaire sur l'emploi, le syndicalisme et le travail, 4(2), 104-124.

Falzon, P. (2013). Pour une ergonomie constructive. Ergonomie constructive, 1-15.

Felio, C. (2014). Le rapport aux TIC des cadres : Réflexions sur l'usage de l'entretien biographique dans une perspective longitudinale. Études de communication, langages, information, médiations, 43, 145-164.

Forté, M., \& Monchatre, S. (2013). Recruter dans l'hôtellerie-restauration : Quelle sélectivité sur un marché du travail en tension? La Revue de l'IRES, 1, 127-150.

Galey, L., Judon, N., Jolly, C., Goutille, F., Morelot, S., Albert, M. Garrigou, A. ; et al. (2019). Proposition méthodologique en ergotoxicologie pour révéler les expositions à des produits chimiques. Activités, 16(1). https://journals.openedition.org/activites/4103

Galiere, S. (2020). Travailler via des plateformes numériques: Une approche en termes d'instruments de gestion. PhD Thesis, Nantes.

Galiere, S. (2021). Vers une instrumentation capacitante des plateformes numériques. Une étude $\mathrm{du}$ cas Airbnb Experience. RIMHE : Revue Interdisciplinaire Management, Homme \& Entreprise, 43(10), 27-52. https://doi.org/10.3917/rimhe.043.0027

Gamkrelidze, T., Barcellini, F., \& Zouinar, M. (2021). Intelligence Artificielle dans les activités professionnelles : Quelles visions des acteurs concernés ? $55^{e}$ congrès de la SELF.

Giacomel, A. (2019). Les enjeux du travail émotionnel individuel et collectif dans les groupes hôteliers multinationaux : La complexité de l'équilibre émotionnel au service de l'homéostasie 
organisationnelle. Thèse de doctorat en sciences de gestion de l'Université d'Angers, dirigée par Dominique Peyrat-Guillard (soutenue le 18 septembre 2019). Mondes du Tourisme, 16.

Girin, J. (1990). L'analyse empirique des situations de gestion : éléments de théorie et de méthodes. In M.C. Martinet (Ed.), Épistémologies et Sciences de Gestion (pp. 141-181). Paris : Éditions Economica.

Glaser, B.G., \& Strauss, A.L. (2010). The Discovery of Grounded Theory: Strategies for Qualitative Research. New Brunswick, NJ : Aldine Transaction.

Gobble, M. M. (2013). Big data: The next big thing in innovation. Research-technology management, 56(1), 64-67.

Gotteland-Agostini, C., Pueyo, V., \& Béguin, P. (2015). Concevoir des cadres pour faire et faire faire : L'activité d'encadrement dans une entreprise horticole. Activités, 12(1) https:// journals.openedition.org/activites/983

Gouédard, C., \& Rabardel, P. (2012). Pouvoir d'agir et capacités d'agir : Une perspective méthodologique ? Illustration dans le champ de la santé, sécurité et conditions de travail. Perspectives interdisciplinaires sur le travail et la santé, 14(2). URL : http://journals.openedition.org/ pistes/2808; https://doi.org/10.4000/pistes.2808

Grefe, G., \& Peyrat-Guillard, D. (2019). Figures de l'emploi touristique : La GRH des mondes de l'hôtellerie et du transport aérien (Vol. 4). ISTE Group.

Grosdemouge, L. (2017). Comprendre le travail des professionnels des ressources humaines pour développer leur pouvoir d'agir : Le cas d'une intervention ergonomique dans un groupe de l'industrie chimique. [PhD Thesis]. Université de Bordeaux.

Grosjean, M., \& Lacoste, M. (1999). Communication et intelligence collective. Le travail à l'hôpital. PUF, Paris.

Guerrier, A. (2016). Régulations et marges de manœuvre situationnelles des encadrants de proximité en sous-traitance: Un enjeu de prévention des TMS. [PhD Thesis]. Université Grenoble Alpes (ComUE).

Guilmot, N., \& Vas, A. (2012). Les cadres intermédiaires au cœur du sensemaking. Revue internationale de psychosociologie et de gestion des comportements organisationnels, 18(45), 77-99.

Havard, C., \& Krohmer, C. (2008). Création et articulation des règles dans le cadre d'un management des compétences. Revue de gestion des ressources humaines, 70, 88-101.

Hellemans, C. \& Vayre, E. (2021). Travail « en débordement » via les technologies : antécédents organisationnels et incidences sur la santé. In : E. Vayre (Ed.), La digitalisation du travail : nouveaux espaces et nouvelles temporalités de travail. Paris : ISTE éditions.

Ivanov, S., \& Webster, C. (2019). Robots, Artificial Intelligence, and Service Automation in Travel, Tourism and Hospitality. Emerald Publishing Limited.

Jauréguiberry, F. (2013). Déconnexion volontaire aux technologies de l'information et de la communication.

Jemine, G. (2017). Déploiement de dispositifs numériques au sein de nouvelles formes d'organisation : De l'émergence à la stabilisation. Sociologies pratiques, 1, 49-59.

Jemine, G. (2019). L'outil face au manager : Le contrôle du travail à l'ère du numérique, un terrain controverse ? Les Cahiers du numérique, 15(4), 137-162.

Jentzsch, C. (2016). Decentralized autonomous organization to automate governance. White paper, November. 
Lamberts, M., Szekér, L., Vandekerckhove, S., Van Gyes, G., Van Hootegem, G., Vereycken, Y., Hansez, I., Mairiaux, P., De Witte, H., \& Bosmans, K. (2016). Jobkwaliteit in België in 2015. Analyse aan de hand van de European Working Conditions Survey EWCS 2015 (Eurofound).

Lanoë, L. (2019). L'organisation contre le management : Le rôle de régulation du manager de proximité et ses empêchements. [PhD Thesis]. Université de Nantes Angers Le Mans.

Le Bossé, Y. (2003). De l'« habilitation » au « pouvoir d'agir » : Vers une appréhension plus circonscrite de la notion d'empowerment. Nouvelles pratiques sociales, 16(2), 30-51.

Lee, M. K., Kusbit, D., Metsky, E., \& Dabbish, L. (2015). Working with machines: The impact of algorithmic and data-driven management on human workers. Proceedings of the 33rd annual ACM conference on human factors in computing systems, 1603-1612.

Leontiev, A. (1984). La théorie de l'activité. Guernier. MC., Guerrier. VD." Interactions.

Leplat, J. (2015). Repères pour l'analyse de l'activité en ergonomie. PUF.

Leplat, J., \& Hoc, J.-M. (1983). Tâche et activité dans l'analyse psychologique des situations. Cahiers de Psychologie Cognitive, 3, 49-63

Lizé, L. (2020). Conditions de travail dans la sous-traitance : Des salariés sous pression un questionnement sur les contours de la segmentation des emplois. CES Working Papers.

Martin, E. (2013). Pourquoi a-t-on encore besoin de managers de proximité ? Une analyse du travail d'encadrement à EDF. La Revue de l'Ires, 76(1), 3-27.

Mayen, P., \& Olry, P. (2012). Expérience du travail et développement pour de jeunes adultes en formation professionnelle. Recherche \& formation, 70, 96-106. https://doi.org/10.4000/ rechercheformation. 1872

Memmi, S., Rosankis, É., Sandret, N., Duprat, P., Léonard, M., Morand, S., \& Tassy, V. (2020). L'évolution des expositions des salariés aux risques professionnels sur les vingt dernières années : Les premiers résultats de l'enquête Sumer 2017. Archives des Maladies Professionnelles et de l'Environnement, 81(1), 69.

Mintzberg, H. (1973). La nature du travail de gestion. New York : Harper \& Row.

Newell, S., \& Marabelli, M. (2015). Strategic opportunities (and challenges) of algorithmic decision-making: A call for action on the long-term societal effects of "datification". The Journal of Strategic Information Systems, 24(1), 3-14.

OECD (2021). "Preparing the Tourism Workforce for the Digital Future". OECD Tourism Papers, No. 2021/02, OECD Publishing, Paris, https://doi.org/10.1787/9258d999-en.

Oiry, E. \& Vignal, J. (2016). Peut-on manager un changement tout en le subissant ? Le cas des managers de proximité. Question(s) de management, 2(2), 61-72.

Oliveri, N., \& Pélissier, N. (2019). Repenser les dispositifs numériques des organisations au prisme des risques technosociaux (rts). Les Cahiers du numérique, 15(4), 87-111.

Petit, J. (2005). Organiser la continuité du service : Intervention sur l'organisation d'une mutuelle de santé. [PhD Thesis]. Université de Bordeaux 2.

Pillai, S. K. B., Kulshreshtha, S. K., \& Korstanje, M. E. (2021). The Real Implications and Effects of Covid19 in the Tourism Industry: What is the future of tourism in a world without tourists? Anais Brasileiros de Estudos Turísticos-ABET.

Piney, C. (2015). Transformation de l'activité d'encadrement de proximité: Que fait le pilotage par la performance à la "proximité" des cadres? Le cas d'une grande administration publique [PhD Thesis]. 
Prost, M., \& Zouinar, M. (2015). De l'hyper-connexion à la déconnexion : Quand les entreprises tentent de réguler l'usage professionnel des e-mails. Perspectives interdisciplinaires sur le travail et la santé, 17(1).

Quérat-Hément, X. (2014). Esprit de service. Passer du marketing au management de l'expérience client. Lexitis Editions.

Reboul, L., Delgoulet, C., Gaudart, C., \& Sutter, S. (2020). La digitalisation de la relation de service : Conséquences sur la santé et sur les parcours des agents de service aux clients d'une compagnie aérienne santé, transformations, transport aérien, activité, parcours. Perspectives interdisciplinaires sur le travail et la santé, 22(1).

Reynaud, J.-D. (1988). Les régulations dans les organisations : Régulation de contrôle et régulation autonome. Revue Française de Sociologie, 29(1), 5-18.

Reynaud, J.-D. (2003). Réflexion : Régulation de contrôle, régulation autonome, régulation conjointe. In La théorie de la régulation sociale de Jean-Daniel Reynaud (pp. 103-116). Paris : La Découverte.

Rocha, R., Mollo, V., \& Daniellou, F. (2017). Le débat sur le travail fondé sur la subsidiarité : Un outil pour développer un environnement capacitant. Activités, 14(2). https:// journals.openedition.org/activites/2999

Rouleau, L. (2016). Micro -Practices of Strategic Sensemaking and Sensegiving: How Middle Managers Interpret and Sell Change Every Day. Journal of Management Studies, 42(7), 1413-1441.

Rouleau, L., \& Balogun, J. (2007). Exploring Middle Managers' Strategic Sensemaking Role in Practice. Advanced Institute of Management Research, 55.

Schmitt, C., Fabbri, R., \& Gallais, M. (2011). Situation de gestion, proximité cognitive et changement dans les organisations. Revue française de gestion, 213(4), 157-169. https://doi.org/ 10.3166/RFG.213.157-169

Srajek, B. (2003). Revalorisation du rôle managérial de l'encadrement de proximité : Cas d'expérimentations. [PhD Thesis]. Lyon 2.

Stein, M.-K., Wagner, E. L., Tierney, P., Newell, S., \& Galliers, R. D. (2019). Datification and the Pursuit of Meaningfulness in Work. Journal of Management Studies, 56(3), 685-717.

Terssac, G. de (2011). Théorie du travail d'organisation. Interpréter l'agir : un défi théorique, 97-121.

Terssac, G. de, \& Cambon, P. (1998). Le travail d'encadrement : relation entre cadre et action. Performances Humaines \& Techniques, HS Septembre, 49-53

Terssac, G. de, Lalande, K., \& Tremblay, D.-G. (2003). Du train à vapeur au TGV : Sociologie du travail d'organisation. Relations Industrielles, 58(1), 147.

Ughetto, P. (2018a). Organiser l'autonomie au travail. Limoges : FYP Éditions.

Ughetto, P. (2018b). Les nouvelles sociologies du travail : Introduction à la sociologie de l'activité. De Boeck Supérieur.

Valiorgue, B. (2016). La fabrique de la RSE par les cadres intermédiaires-Le cas Adecco. Revue française de gestion, 42(256), 19-39.

Valléry, G., \& Leduc, S. (2005). Contribution ergonomique à l'analyse des relations de service exemple de professionnalisation d'une fonction d'accueil en bureau de poste. Le Travail Humain, 68(2), 153-189. 
Weick, K.E. (1993). Sensemaking in Organizations: Small Structures with Large Consequences. In J. K. Murnigham (Ed.), Social Psychology in Organizations: Advances in Theory and Research. Englewood Cliffs, NJ : Prentice Hall.

Wolff, L. (2013). Un encadrement de plus en plus... encadré : le blues des managers. Santé et travail (Paris), 82, 30-32.

Yatchinovsky, A. (2018). L'approche systémique : Pour gérer l'incertitude et la complexité. ESF Sciences Humaines.

Yin, R. K. (2009). Case study research: Design and methods. In L. Bickman \& D. J. Rog (Eds.), Essential guide to qualitative methods in organizational research, 5(5). Thousand Oaks, CA : Sage Publications.

\section{NOTES}

1. Dans cet article le terme manager de réception et manager de proximité (MDP) sont utilisés de manière identique.

2. L'empowerment tel qu'il est défini ici par l'auteure désigne les formes d'action concrètes mises en œuvre par les instances d'encadrement et de régulation (management, politiques d'entreprise) pour permettre de «renforcer ou acquérir du pouvoir» des salariés. Ce terme évoque « une habilitation, quelquefois prédéfinie ou délimitée, permettant à un collaborateur ou à une équipe auxquels on en donne les moyens de prendre des initiatives et des décisions dans leur périmètre d'actions et possiblement en dehors de leurs descriptions de postes ».

3. Le concept de situation est défini comme «ce avec quoi une personne est amenée à agir » (Duhamel, Olry, \& Cerf, 2021, p. 1-2).

4. Le pouvoir d'agir tel que nous le décrivons dépend «des conditions externes et internes au sujet, qui sont réunies à un moment particulier, comme l'état fonctionnel du sujet, les artefacts et ressources disponibles, les occasions d'intervention, etc. Il est toujours situé dans un rapport singulier au monde réel, rapport qui actualise et réalise la capacité d'agir en transformant les potentialités en pouvoir » (Gouédard \& Rabardel, 2012).

5. Progiciel de gestion intégré (PGI) ou plus couramment en anglais enterprise ressource planning (ERP).

6. Méthode de management plus couramment appelée yield management issue du transport aérien.

7. Dispositif de gestion de la relation client (GRC) ou customer relationship management (CRM).

8. L'asset light consiste à se libérer des charges de propriété en valorisant une croissance du parc hôtelier par les contrats en management et les franchises (Grefe \& Peyrat-Guillard, 2019)

9. L'étude de cas prend le sens ici d'une "recherche empirique qui étudie un phénomène contemporain dans un contexte réel, lorsque les frontières entre le phénomène et le contexte n'apparaissent pas clairement, et dans laquelle on mobilise des sources empiriques multiples » (Yin, 2009, p. 18).

10. Il s'agit du terme créé pour faire référence à la chaîne hôtelière étudiée.

11. L'overbooking consiste à prendre un nombre de réservations supérieures au nombre de places disponibles dans l'hôtel

12. Les planneurs sont les acteurs qui gravitent autour de l'activité de travail des MDP sans en être directement des supérieurs hiérarchiques (chef de projet IT, etc.).

13. On peut définir l'activité de pilote ou le pilotage comme l'activité qui, dans l'espace de la production, consiste à faire faire. 
14. Le terme shift est utilisé en hôtellerie pour décrire ce que l'on qualifie comme la période du poste dans le cadre du travail posté.

15. Le partage attentionnel a été calculé sur base des enregistrements vidéo et de l'identification des mouvements de tête perceptibles entre le smartphone et le client.

16. KPI est le terme utilisé par les MDP pour désigner les indicateurs clés de performance utilisés.

\section{RÉSUMÉS}

L'article explore l'activité du manager de proximité (MDP) dans le cadre de la digitalisation dans trois hôtels d'une chaîne hôtelière internationale. La méthodologie permet une analyse de l'activité du manager sur deux niveaux: l'activité du MDP dans son rôle de gestionnaire au quotidien en environnement digitalisé et l'activité du MDP dans l'accompagnement d'un projet pilote de digitalisation de l'accueil via le smartphone. L'analyse ergonomique montre un effet controversé des technologies avec le renforcement du cadre gestionnaire et du contrôle sur l'activité managériale. L'analyse ergonomique est également révélatrice du rôle de régulation du MDP. En contexte de changement du mode d'accueil, les résultats révèlent des tentatives d' «empowerment» du MDP pour rendre acceptable le smartphone par les réceptionnistes à travers des mises en situations destinées au développement du travail émotionnel des réceptionnistes. Ce cadre gestionnaire n'offre pas la possibilité au MDP d'être en capacité d'agir pour le développement d'un réel pouvoir d'agir des réceptionnistes avec le dispositif. Des pistes sont énoncées afin d'envisager une digitalisation source de développement de l'activité. Une d'entre elles repose sur le rapprochement des concepteurs et des usagers: managers, réceptionnistes, revenue manager autour de l'objet de la personnalisation du service. Une autre piste consiste à penser la future organisation du travail avec la technologie en amont de la conception, à travers la réalisation de simulation dynamique prenant en compte l'activité réelle à la réception.

The article explores the activity of the front office manager (FOM) in the context of the digitalization in three hotels of an international hotel chain. The methodology allowed us to analyze the FOM's activity on two levels: his activity as part of his daily management duties in a digitized environment and his activity in the accompaniment of a pilot reception digitalization project via smartphone. The ergonomic analysis indicates a controversial effect of technologies with the reinforcement of the managerial framework and of control of the managerial activity. The ergonomic analysis also reveals the regulatory role of the FOM. In the context of a change in the reception mode, the results reveal attempts to 'empower' the FOM to make the smartphone acceptable to the receptionists through situations designed to develop the receptionists' emotional work. This managerial framework does not allow the FOM to be in a position to take steps to develop real power for the receptionists to act with the device. Certain suggestions are made to consider digitalization as a source of activity development. One of these is based on bringing designers and users (managers, receptionists and revenue managers) together around the objective of personalizing the service. Another approach is to think about the future organization of work with technology upstream of the design phase, through the creation of dynamic simulations that take into account the real activity at the reception desk. 
INDEX

Keywords : tourism, digitalisation, management, ergonomics, empowerment

Mots-clés : tourisme, digitalisation, management, ergonomie, pouvoir d'agir

\section{AUTEURS}

\section{PIERRE FLANDRIN}

Université libre de Bruxelles (ULB), Centre de recherche en psychologie du travail et de la consommation (CRPsyTC), Av. Roosevelt, 50 (CP122), 1050 Bruxelles, Belgique -

Pierre.Flandrin@ulb.be

\section{CÉCILE VAN DE LEEMPUT}

Université libre de Bruxelles (ULB), Centre de recherche en psychologie du travail et de la consommation (CRPsyTC), Av. Roosevelt, 50 (CP122), 1050 Bruxelles, Belgique

\section{CATHERINE HELLEMANS}

Université libre de Bruxelles (ULB), Centre de recherche en psychologie du travail et de la consommation (CRPsyTC), Av. Roosevelt, 50 (CP122), 1050 Bruxelles, Belgique 